Supporting Information 


\title{
In silico driven design and synthesis of rhodanine derivatives as novel antibacterials targeting the enoyl reductase InhA
}

\author{
Liudas Slepikas, ${ }^{a, b \dagger}$ Gianpaolo Chiriano, ${ }^{a \dagger}$ Remo Perozzo, ${ }^{a}$ Sébastien Tardy, ${ }^{a}$ Agata Kranjc, ${ }^{a}$ \\ Ophélie Patthey-Vuadens, ${ }^{a}$ Hajer Ouertatani-Sakouhi, ${ }^{c}$ Sébastien Kicka, ${ }^{d}$ Christopher F. \\ Harrison, ${ }^{e}$ Tiziana Scrignari, ${ }^{f}$ Karl Perron, ${ }^{f}$ Hubert Hilbi, ${ }^{e, g}$ Thierry Soldati, ${ }^{d}$ Pierre Cosson, ${ }^{c}$ \\ Eduardas Tarasevicius ${ }^{b}$ and Leonardo Scapozza $a^{{ }^{*}}$
}

${ }^{a}$ School of Pharmaceutical Sciences, Department of Pharmaceutical Biochemistry, University of Geneva and University of Lausanne, 30 Quai Ernest Ansermet, 1211 Geneva, Switzerland; ${ }^{\mathrm{b}}$ Faculty of Pharmacy, Lithuanian University of Health Sciences, LT 44307 Kaunas, Lithuania; ${ }^{\mathrm{c} D e p a r t m e n t}$ of cell physiology and metabolism, CMU, 1 Rue Michel-Servet, 1211 Geneva, Switzerland; 'Department of Biochemistry, University of Geneva, 30 Quai Ernest Ansermet, 1211 Geneva, Switzerland; ${ }^{\mathrm{e}}$ Max von Pettenkofer Institute, Department of Medicine, LudwigMaximilians University Munich, 80336 Munich, Germany; ${ }^{f}$ Microbiology Unit, Department of Botany and Plant Biology, University of Geneva, Switzerland; Institute of Medical Microbiology, Department of Medicine, University of Zürich, Gloriastrasse 30/32, 8006 Zürich, Switzerland.

\footnotetext{
${ }^{\dagger}$ These authors contributed equally

*Author correspondence to Prof. Leonardo Scapozza: +41223793363 and leonardo.scapozza@unige.ch
} 


\section{List of contents:}

- Figure $\mathrm{S} 1$. The $\mathrm{IC}_{50}$ of triclosan towards $M t b \operatorname{InhA}$

- Table S1. Inhibitory activity and $\mathrm{IC}_{50}$ of derivatives 1-34 towards $M t b$ InhA

- $\quad$ Table S2. In vitro antibacterial activity of compounds 1-34

- HPLC analysis for 1-34: methods and chromatograms

- References SI 


\section{Figure S1}

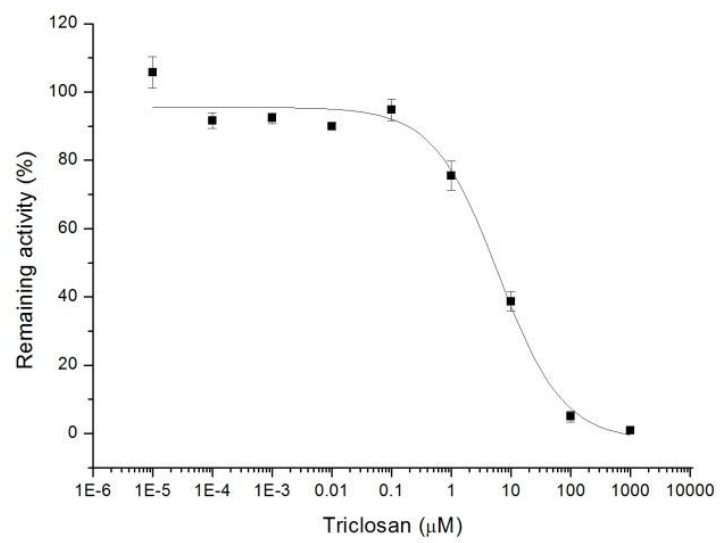

Figure S1. The $\mathrm{IC}_{50}$ of triclosan towards $M t b$ InhA. Each data point represents the mean of three independent experiments. The percent activity thus calculated was plotted against log concentration of the compound. The data were analyzed by nonlinear regression and the $\mathrm{IC}_{50}$ values determined from the fit of the data. The determined $\mathrm{IC}_{50}$ values for triclosan are very close to those described in the literature. ${ }^{1}$ 
Table S1. Inhibitory activity and $\mathrm{IC}_{50}$ of derivatives 1-34 towards $M t b \operatorname{Inh} \mathrm{A}$

\begin{tabular}{|c|c|c|}
\hline Compounds & Remaining $M t b \operatorname{InhA}$ activity $(\%)^{\Delta}$ & $M t b \operatorname{Inh} A I_{50}(\mu M)$ \\
\hline 1 & $93.6 \pm 4.3$ & - \\
\hline 2 & $81.6 \pm 4.6$ & - \\
\hline 3 & $79.0 \pm 3.3$ & - \\
\hline 4 & $70.1 \pm 3.1$ & - \\
\hline 5 & $69.7 \pm 2.4$ & - \\
\hline 6 & $76.9 \pm 3.0$ & - \\
\hline 7 & $82.6 \pm 0.7$ & - \\
\hline 8 & $69.5 \pm 2.2$ & - \\
\hline 9 & $96.6 \pm 4.3$ & - \\
\hline 10 & $104.3 \pm 3.5$ & - \\
\hline 11 & $87.4 \pm 3.0$ & - \\
\hline 12 & $21.7 \pm 3.4$ & $28.6 \pm 1.3$ \\
\hline 13 & $52.6 \pm 3.0$ & - \\
\hline 14 & $21.9 \pm 1.5$ & - \\
\hline 15 & $98.1 \pm 3.9$ & - \\
\hline 16 & $6.9 \pm 1.2$ & $24.4 \pm 0.3$ \\
\hline 17 & Total inhibition & $19.1 \pm 1.3$ \\
\hline 18 & $35.7 \pm 3.8$ & $19.1 \pm 1.3$ \\
\hline 19 & $99.9 \pm 2.1$ & - \\
\hline
\end{tabular}




\begin{tabular}{|c|c|c|}
\hline 20 & $77.4 \pm 2.1$ & - \\
\hline 21 & Total inhibition & $22.4 \pm 1.5$ \\
\hline 22 & $79.8 \pm 2.3$ & - \\
\hline 23 & $103.7 \pm 3.8$ & - \\
\hline 24 & $6.7 \pm 2.7$ & $14.7 \pm 2.2$ \\
\hline 25 & Total inhibition & $13.0 \pm 2.1$ \\
\hline 26 & Total inhibition & $12.2 \pm 1.6$ \\
\hline 27 & Total inhibition & $13.0 \pm 2.1$ \\
\hline 28 & Total inhibition & $15.2 \pm 2.4$ \\
\hline 29 & Total inhibition & $19.7 \pm 1.4$ \\
\hline 30 & Total inhibition & $9.5 \pm 2.2$ \\
\hline 31 & $70.5 \pm 18.7^{*}$ & $\sim 38 *$ \\
\hline 32 & Total inhibition & $2.9 \pm 0.7$ \\
\hline 33 & Total inhibition & $8.7 \pm 0.5$ \\
\hline 34 & Total inhibition & $11.0 \pm 0.7$ \\
\hline Triclosan & $20.2 \pm 4.5$ & $6.1 \pm 2.1$ \\
\hline
\end{tabular}

${ }^{\Delta}$ Remaining Mtb InhA activity determined at $30 \mu \mathrm{M}$ concentration of compounds.

*Very poor solubility giving interference during measurement.

“_": not determined. 
Table S2. In vitro antibacterial activity of compounds 1-34

\begin{tabular}{|c|c|c|c|}
\hline Compounds & $\begin{array}{l}\text { Mm anti-virulence } \\
\text { evaluation of } \\
\text { phagocytic plaque } \\
\text { formation }\end{array}$ & $\begin{array}{c}\text { Normalized } \\
\text { Extracellular } L p \\
\text { replication }^{\text {b }} \\
\text { (Antibiotic assay) }\end{array}$ & $\begin{array}{c}\text { Normalized } \\
\text { Intracellular } L p \\
\text { replication }^{\text {b }} \\
\text { (Infection assay) }\end{array}$ \\
\hline 1 & 0 & $0.03 \pm 0.05$ & $1.00 \pm 0.07$ \\
\hline 2 & 0 & $0.05 \pm 0.04$ & $0.98 \pm 0.07$ \\
\hline 3 & 0 & $0.06 \pm 0.04$ & $1.11 \pm 0.08$ \\
\hline 4 & 0 & $0.02 \pm 0.02$ & $1.25 \pm 0.06$ \\
\hline 5 & 0 & $0.002 \pm 0.04$ & $1.14 \pm 0.12$ \\
\hline 6 & 0 & $0.06 \pm 0.04$ & $1.15 \pm 0.04$ \\
\hline 7 & 0 & $0.05 \pm 0.04$ & $1.12 \pm 0.04$ \\
\hline 8 & 0 & $0.06 \pm 0.05$ & $1.14 \pm 0.13$ \\
\hline 9 & 0 & $0.06 \pm 0.03$ & $1.08 \pm 0.15$ \\
\hline 10 & 0 & $0.68 \pm 0.12$ & $1.05 \pm 0.09$ \\
\hline 11 & 0 & $0.02 \pm 0.04$ & $1.00 \pm 0.04$ \\
\hline 12 & 0 & $0.20 \pm 0.25$ & $0.92 \pm 0.07$ \\
\hline 13 & 1 & $0.06 \pm 0.04$ & $0.82 \pm 0.03$ \\
\hline 14 & 0 & $0.06 \pm 0.04$ & $0.90 \pm 0.07$ \\
\hline 15 & 2 & $0.04 \pm 0.05$ & $0.87 \pm 0.07$ \\
\hline 16 & 0 & $0.005 \pm 0.05$ & $0.84 \pm 0.10$ \\
\hline 17 & 4 & $0.01 \pm 0.01$ & $0.90 \pm 0.05$ \\
\hline
\end{tabular}




\begin{tabular}{|c|c|c|c|}
\hline 18 & 1 & $0.39 \pm 0.11$ & $0.90 \pm 0.04$ \\
\hline 19 & 0 & $0.81 \pm 0.24$ & $1.01 \pm 0.05$ \\
\hline 20 & 0 & $0.23 \pm 0.32$ & $0.95 \pm 0.04$ \\
\hline 21 & 4 & $0.05 \pm 0.05$ & $1.04 \pm 0.18$ \\
\hline 22 & 1 & $0.05 \pm 0.04$ & $0.79 \pm 0.12$ \\
\hline 23 & 2 & $0.05 \pm 0.04$ & $1.06 \pm 0.12$ \\
\hline 24 & 2 & $0.09 \pm 0.13$ & $0.92 \pm 0.20$ \\
\hline 25 & 3 & $0.18 \pm 0.24$ & $0.25 \pm 0.08$ \\
\hline 26 & 3 & $0.18 \pm 0.11$ & $0.94 \pm 0.10$ \\
\hline 27 & 3 & $0.06 \pm 0.12$ & $0.39 \pm 0.11$ \\
\hline 28 & 4 & $0.45 \pm 0.29$ & $0.80 \pm 0.06$ \\
\hline 29 & 4 & $0.02 \pm 0.04$ & $0.23 \pm 0.07$ \\
\hline 30 & 4 & $0.005 \pm 0.06$ & $0.48 \pm 0.19$ \\
\hline 31 & 0. & $0.50 \pm 0.15$ & $0.94 \pm 0.09$ \\
\hline 32 & 4 & $0.06 \pm 0.04$ & $0.97 \pm 0.11$ \\
\hline 33 & 4 & $0.05 \pm 0.04$ & $0.11 \pm 0.04$ \\
\hline 34 & 4 & $0.04 \pm 0.05$ & $0.30 \pm 0.06$ \\
\hline
\end{tabular}

a "__" means not determined

b : Compounds have been tested at $30 \mu \mathrm{M}$ 


\section{HPLC analysis for 1-34: methods and chromatograms}

$1 \mathrm{mg}$ of each compound was weighed and dissolved in $50 \mathrm{ml}$ of acetonitrile. The sample was filtered through a filter and placed into auto-sampler vial for analysis. HPLC analysis was performed on Agilent 1200 series with Agilent Zorbax RRHD column C18 column (1.8 $\mu \mathrm{m}, 2.1$ $\mathrm{mm} \times 50 \mathrm{~mm}$ ) applying a flow rate of $0.4 \mathrm{~mL} / \mathrm{min}$ and an injection volume of $1 \mu \mathrm{L}$. For derivatives 1-10, 13-17, 21-34, the mobile phase was composed of solution A $(0.1 \%$ formic acid in water) and solution B (acetonitrile), and the following gradient was applied: 0 min 100\% of A; 3-4 $\min 100 \%$ of B; 5 min 100\% of A. For derivatives 12-13, 18-20, different gradient conditions were applied: $0 \min 100 \%$ of $\mathrm{A} ; 3 \min 10 \%$ of $\mathrm{A} ; 90 \% \mathrm{~B} ; 6 \min 100 \%$ of $\mathrm{B} ; 8 \mathrm{~min} 100 \%$ of $\mathrm{B}$, $10 \min 100 \%$ of $\mathrm{A}$. For derivatives $\mathbf{1 - 1 0}, \mathbf{1 8 - 2 0}$ and $\mathbf{2 4}$, detection was performed at the wavelength of $474 \mathrm{~nm}$, whereas for derivatives 11-17, 21-23, 25-34 at the wavelength of $410 \mathrm{~nm}$. In all chromatograms here below reported, the y-axis represents the absorption expressed in $\mathrm{mAU}$ and $\mathrm{x}$-axis represents time in minutes. The major peak, identified within the retention time ranging from $5 \mathrm{~min}$ to $7 \mathrm{~min}$, corresponds to the $\mathrm{Z}$ isomer, whereas the second peak as well as the shoulder present in some of the chromatograms is not an impurity but the isomer E due to the double bond present between the rhodanine ring and arylidene fragment at the position 5. This has been revealed by additional high-resolution mass spectral analysis (qTOF). This is in line with ${ }^{1} \mathrm{H}-\mathrm{NMR}$ experiments showing the $\mathrm{E} / \mathrm{Z}$ isomerism. 
Characteristic base-line drift due to the mobile phase gradient formation, when acetronitrile goes from $0 \%$ to $90-110 \%$, causing a change in the absorption.

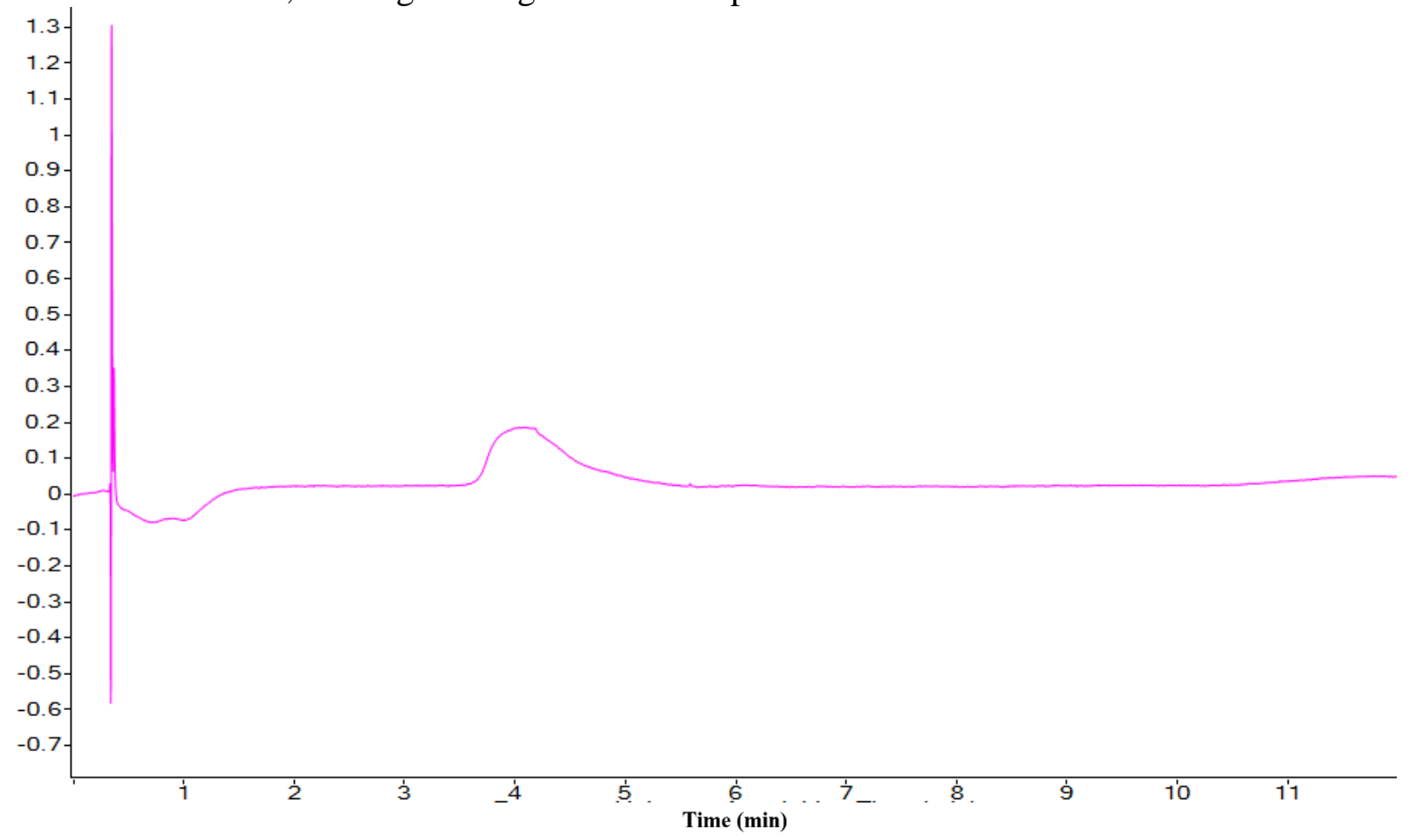

(2S)-2-[(5Z)-5-\{[4-(dimethylamino)phenyl]methylidene\}-4-oxo-2-sulfanylidene-1,3thiazolidin-3-yl]propanoic acid (1).

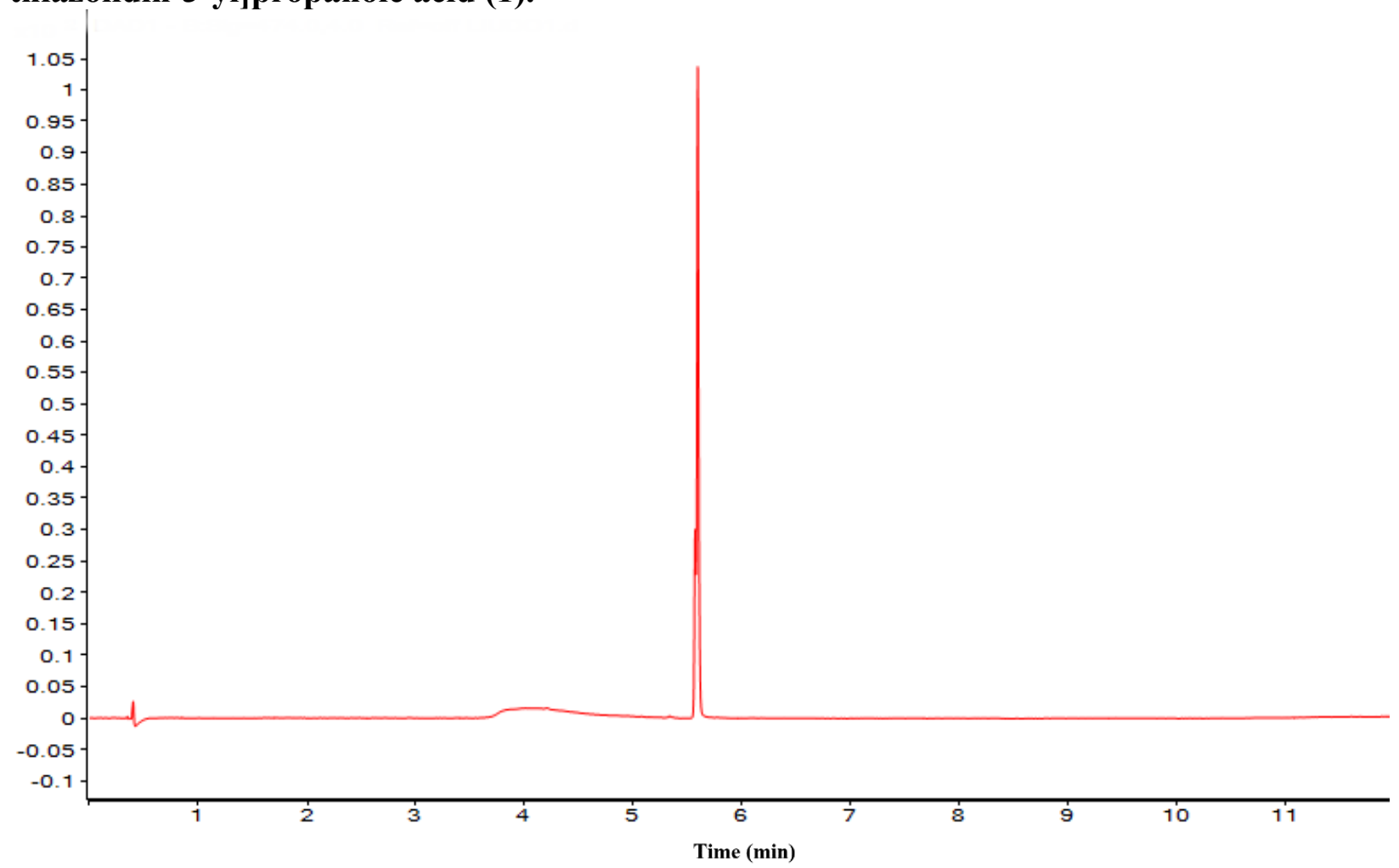


(2R,S)-\{(5Z)-5-[4-(dimethylamino)benzylidene]-4-oxo-2-thioxo-1,3-thiazolidin-3yl\}butanoic acid (2).

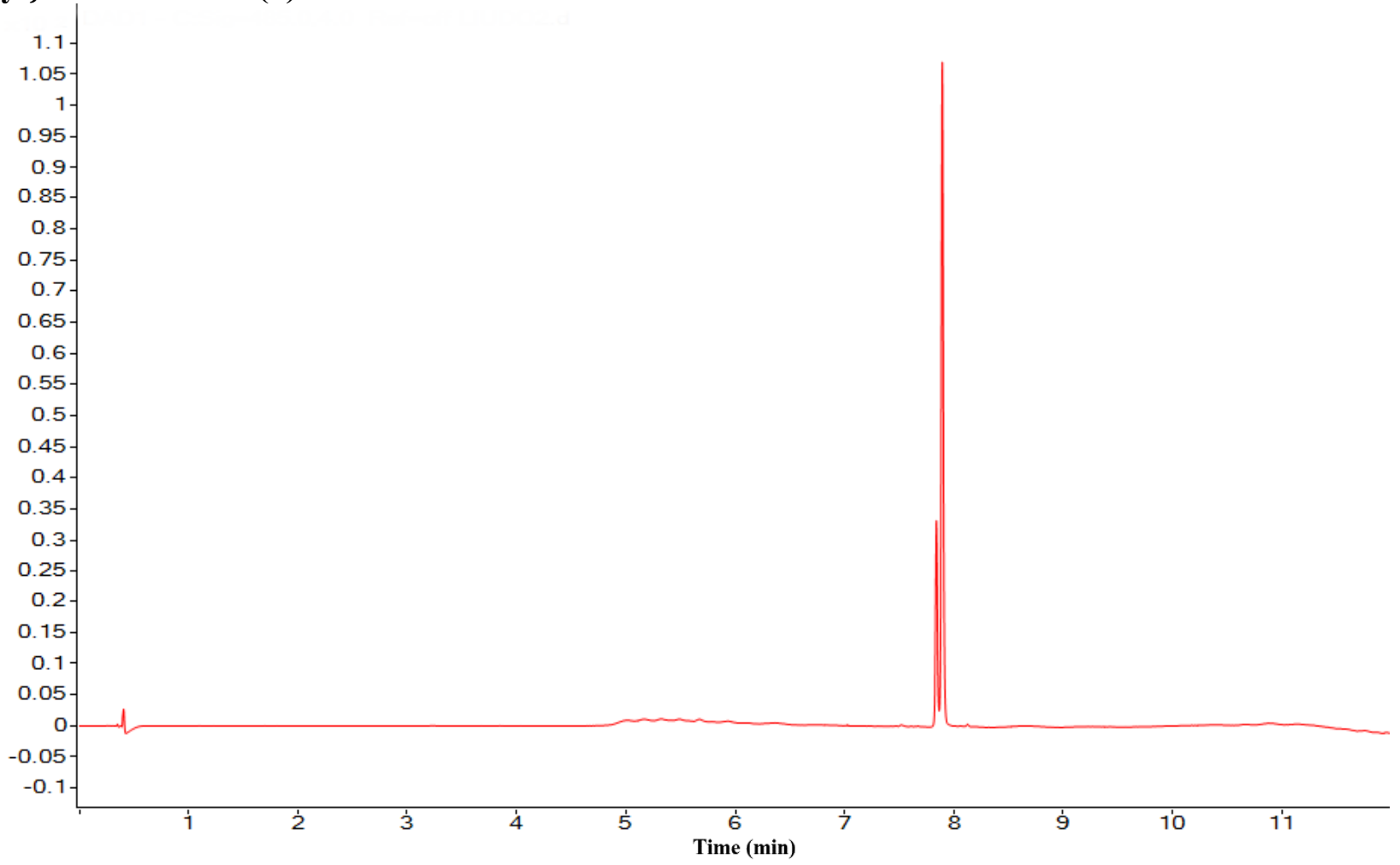

(2R,S)-\{(5Z)-5-[4-(dimethylamino)benzylidene]-4-oxo-2-thioxo-1,3-thiazolidin-3-yl\}-3methylbutanoic acid (3).

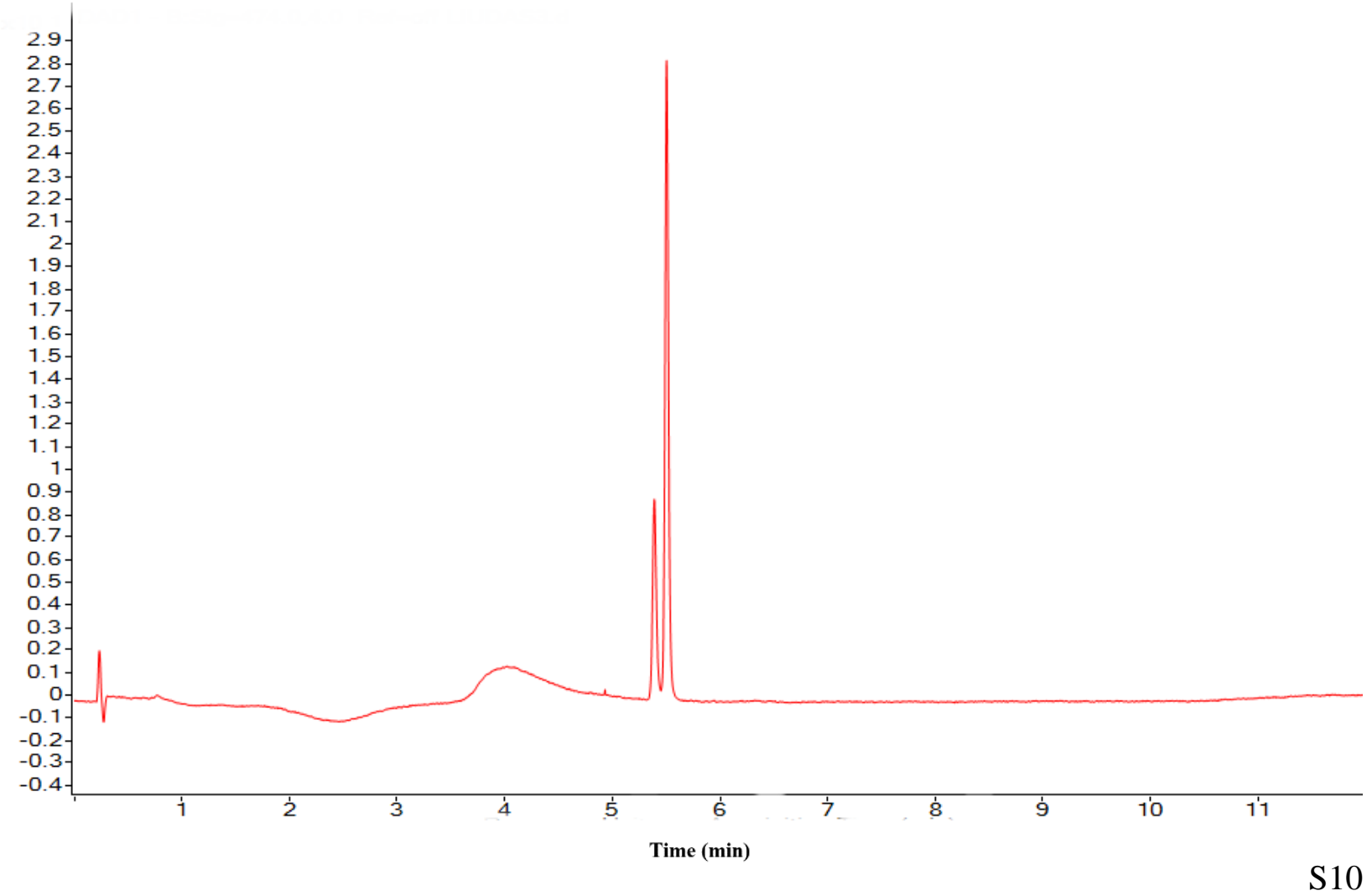


(2R)-2-\{(5Z)-5-[4-(dimethylamino)benzylidene]-4-oxo-2-thioxo-1,3-thiazolidin-3-yl\}-4methylpentanoic acid (4).

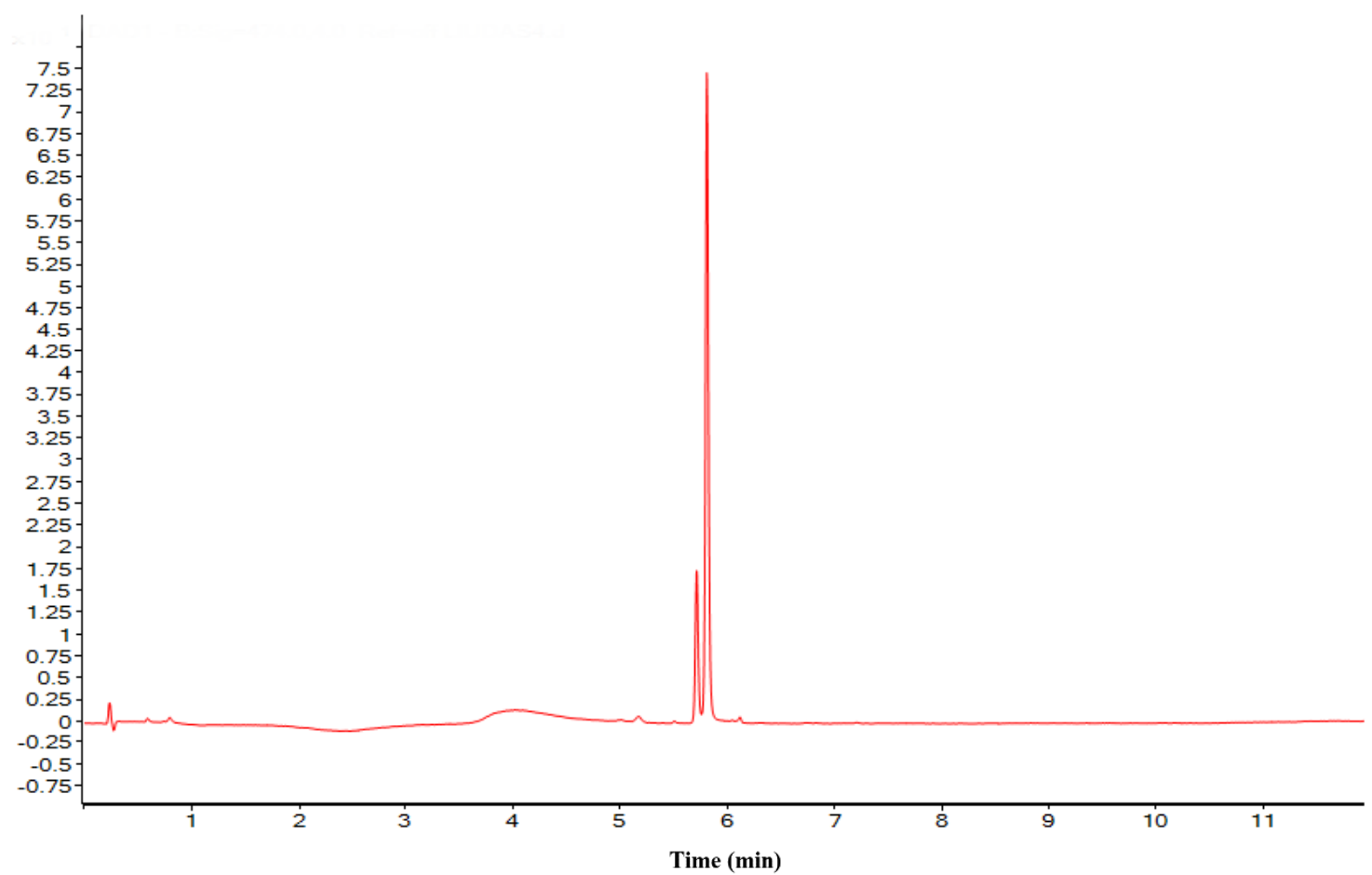

(2S)-2-\{(5Z)-5-[4-(dimethylamino)benzylidene]-4-oxo-2-thioxo-1,3-thiazolidin-3-yl\}-4methylpentanoic acid (5).

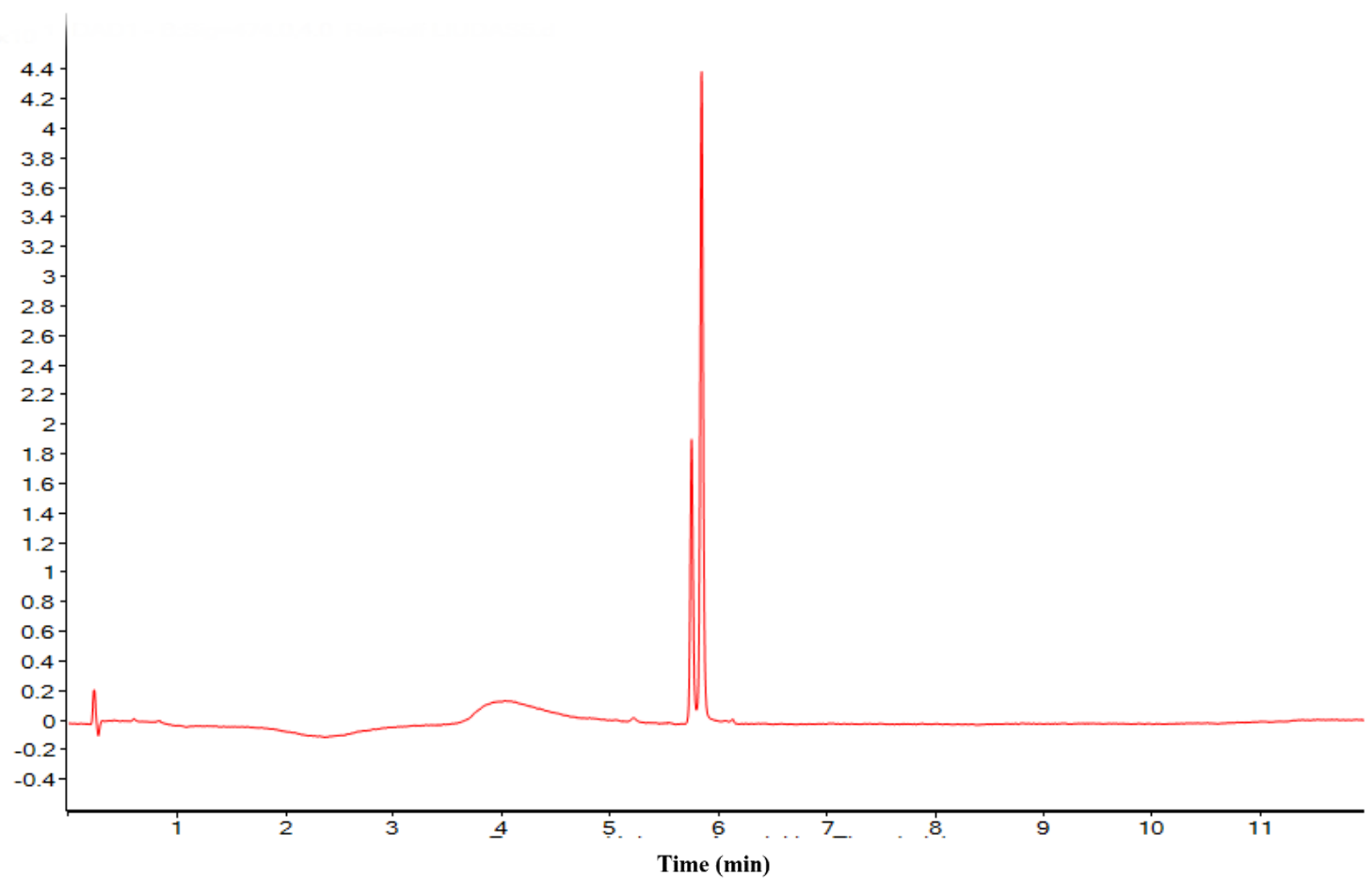


(2S)-2-\{(5Z)-5-[4-(dimethylamino)benzylidene]-4-oxo-2-thioxo-1,3-thiazolidin-3-yl\}-3methylpentanoic acid (6).

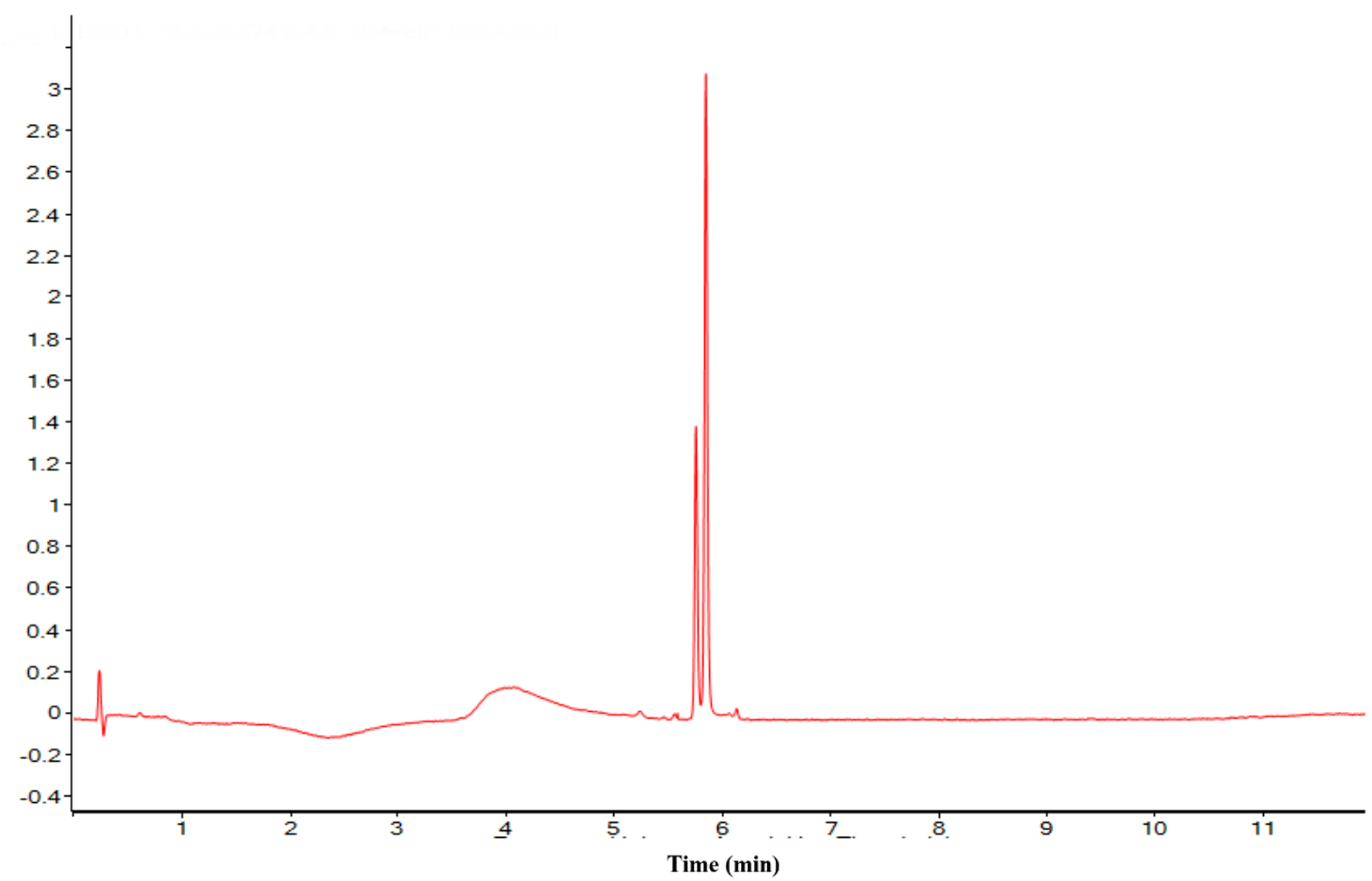

(2R,S)—\{(5Z)-5-[4-(dimethylamino)benzylidene]-4-oxo-2-thioxo-1,3-thiazolidin-3yl\}pentanoic acid (7).

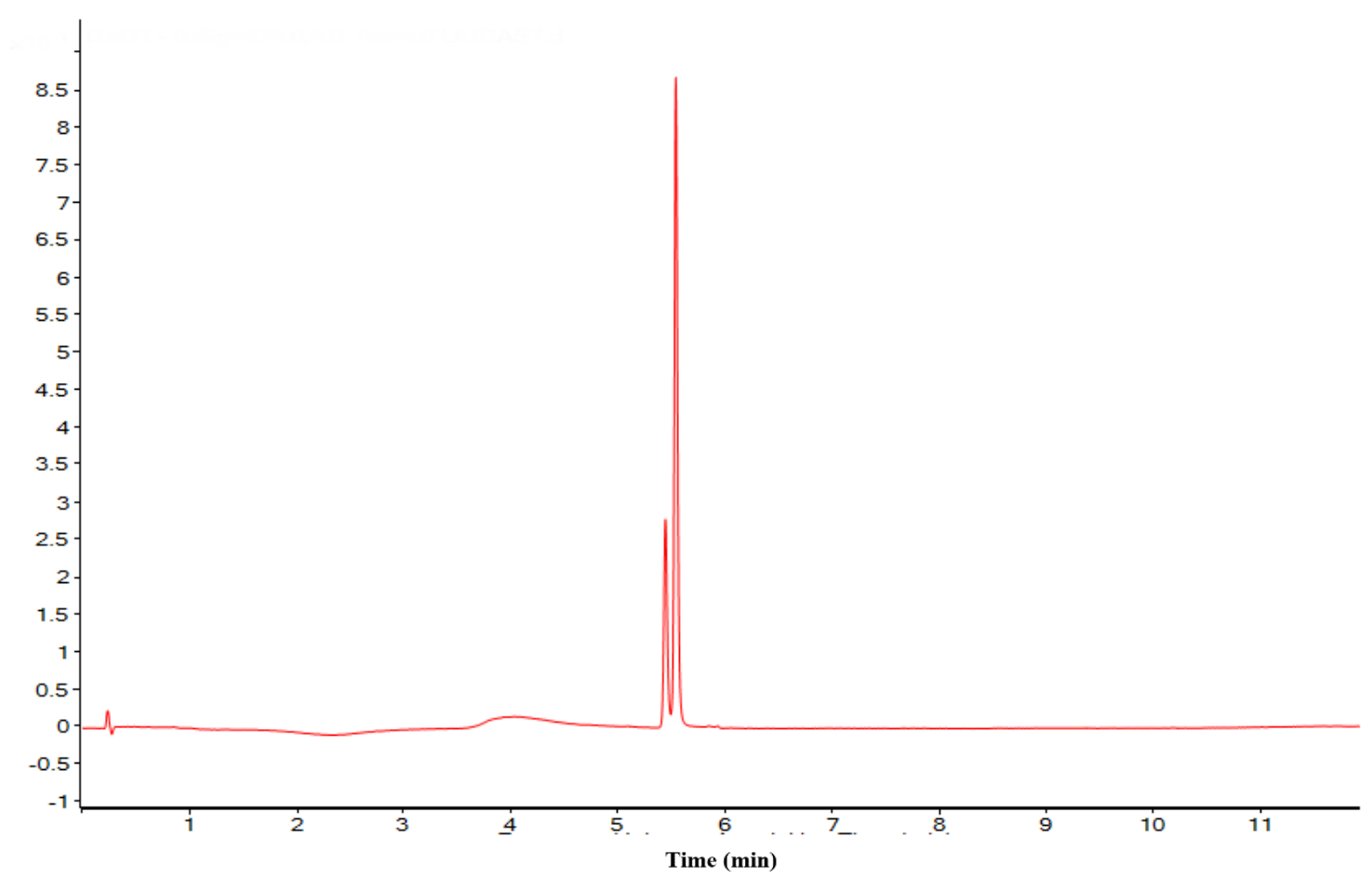


(2R,S)—\{(5Z)-5-[4-(dimethylamino)benzylidene]-4-oxo-2-thioxo-1,3-thiazolidin-3yl\}hexanoic acid (8).

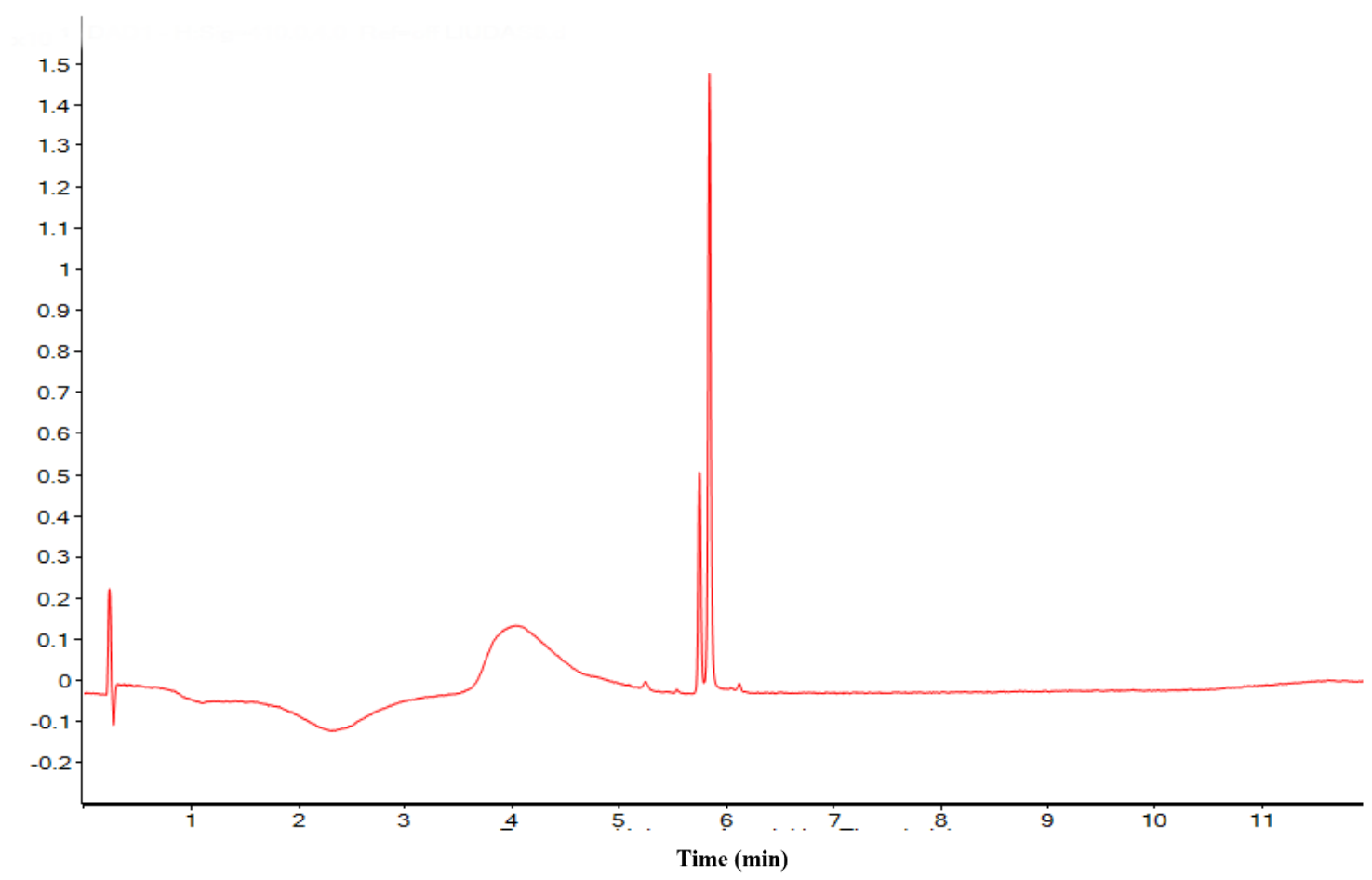

(2R,S)-\{(5Z)-5-[4-(dimethylamino)benzylidene]-4-oxo-2-thioxo-1,3-thiazolidin-3-yl\}-4(methylsulfanyl)butanoic acid (9).

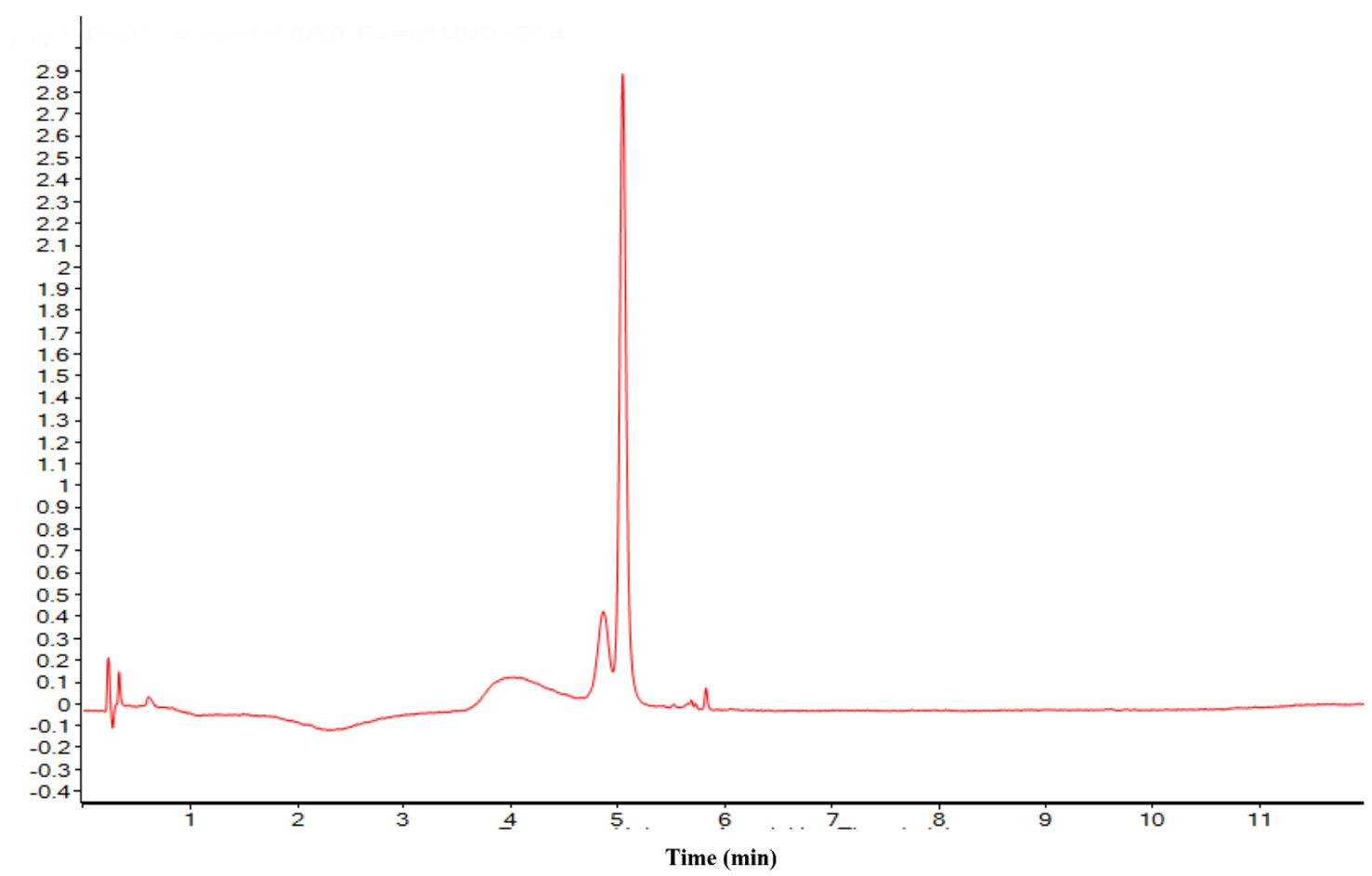


(2S)-2-\{(5Z)-5-[4-(dimethylamino)benzylidene]-4-oxo-2-thioxo-1,3-thiazolidin-3-yl\}-4(methylselenyl)butanoic acid (10).

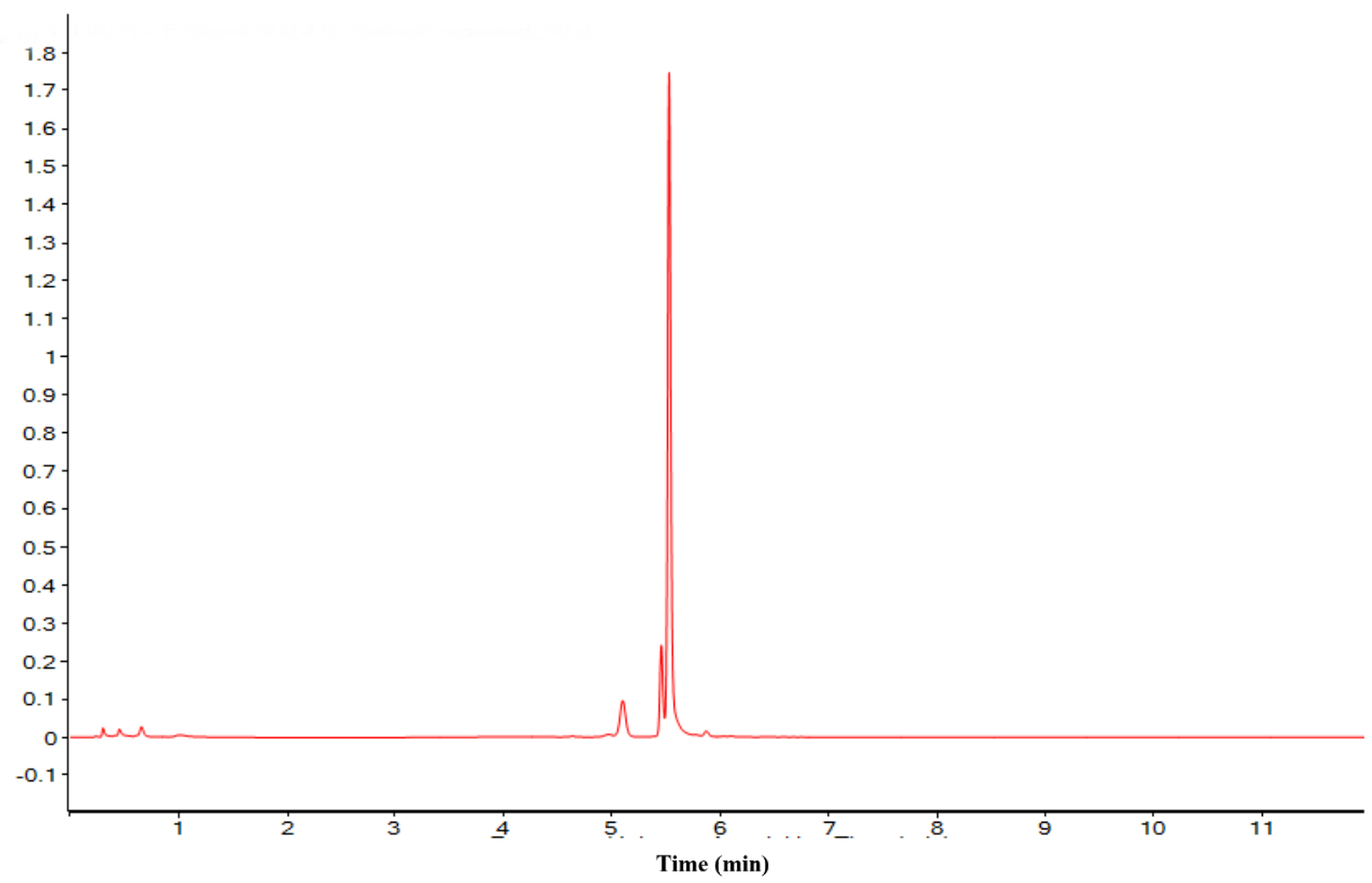

(2S)-2-\{(5Z)-5-[4-(dimethylamino)benzylidene]-4-oxo-2-thioxo-1,3-thiazolidin-3yl butanedioic acid (11).

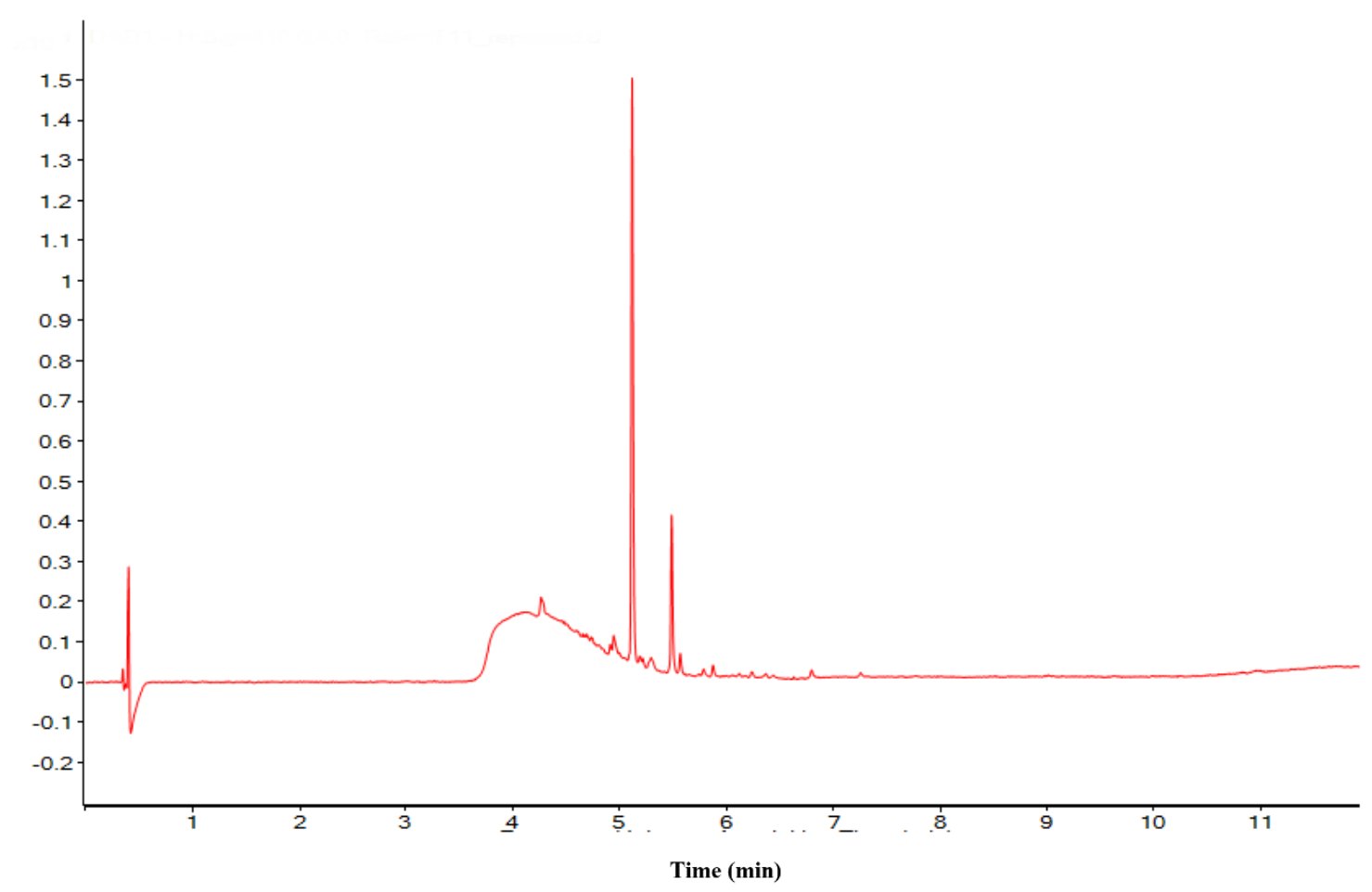


(2S)-2-[(5Z)- 5-[4-(dimethylamino)benzylidene]-4-oxo-2-thioxo-1,3-thiazolidin-3-yl]-3-(1Hindol-3-yl)propanoic acid (12).

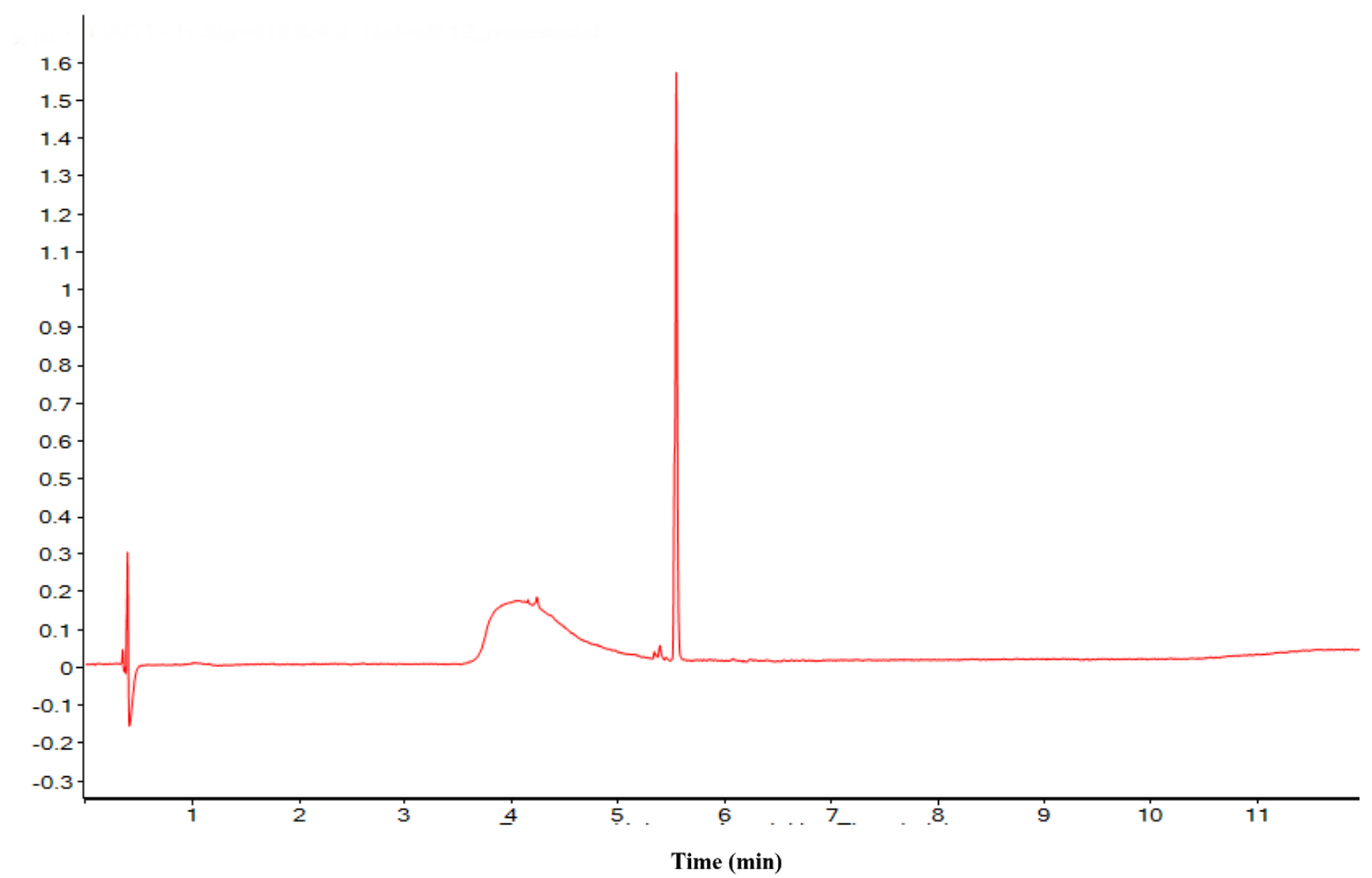

(2S)-4-methyl-2-[(5Z)-5-\{[4-(dimethylamino)naphthalene-1-yl]methylidene $\}-4-0 x 0-2-$ thioxo-1,3-thiazolidin-3-yl]pentanoic acid (13).

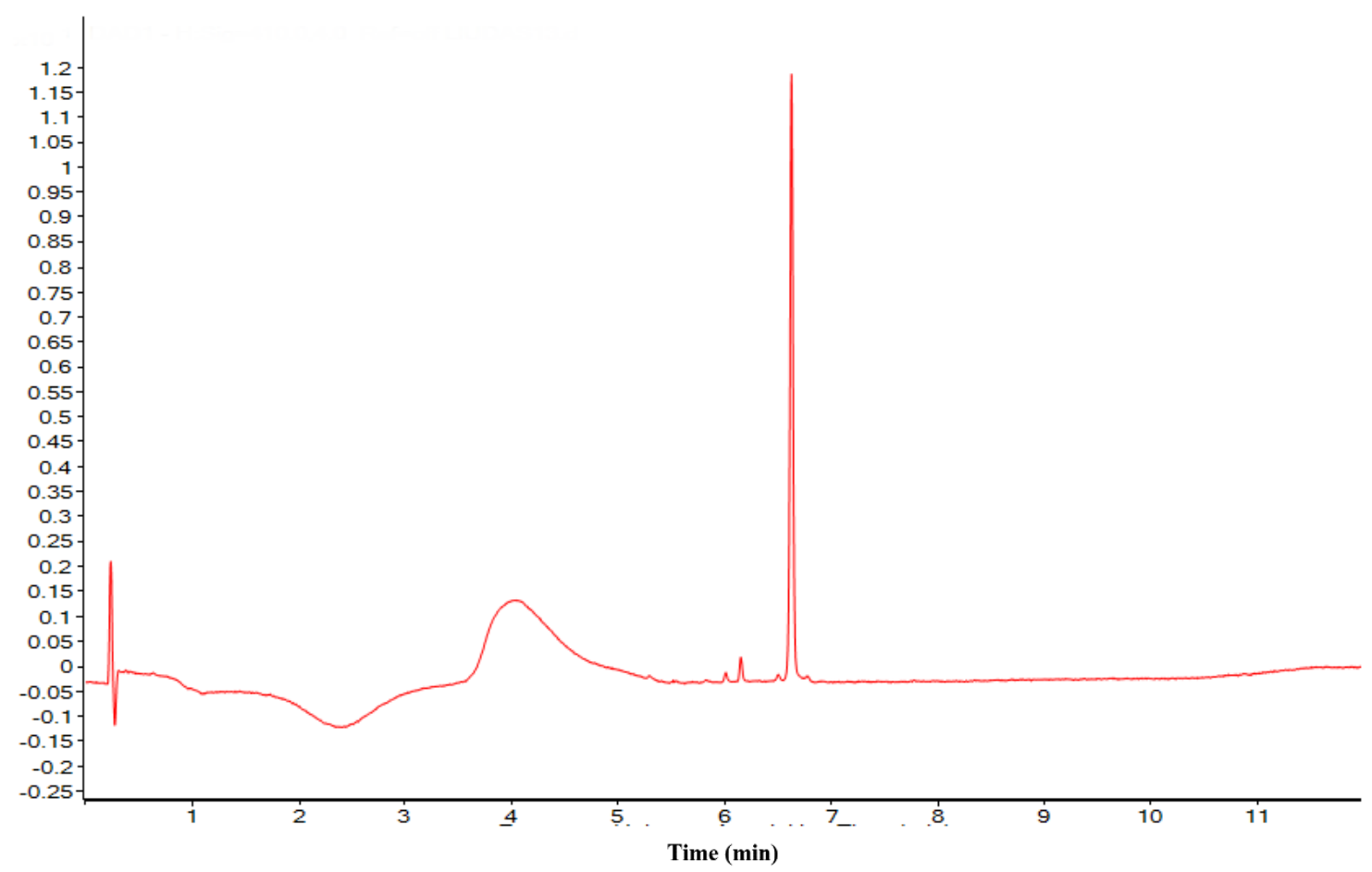


(2R)-4-methyl-2-[(5Z)-5-\{[4-(dimethylamino)naphthalene-1-yl]methylidene $\}-4-0 x 0-2-$ thioxo-1,3-thiazolidin-3-yl]pentanoic acid (14).

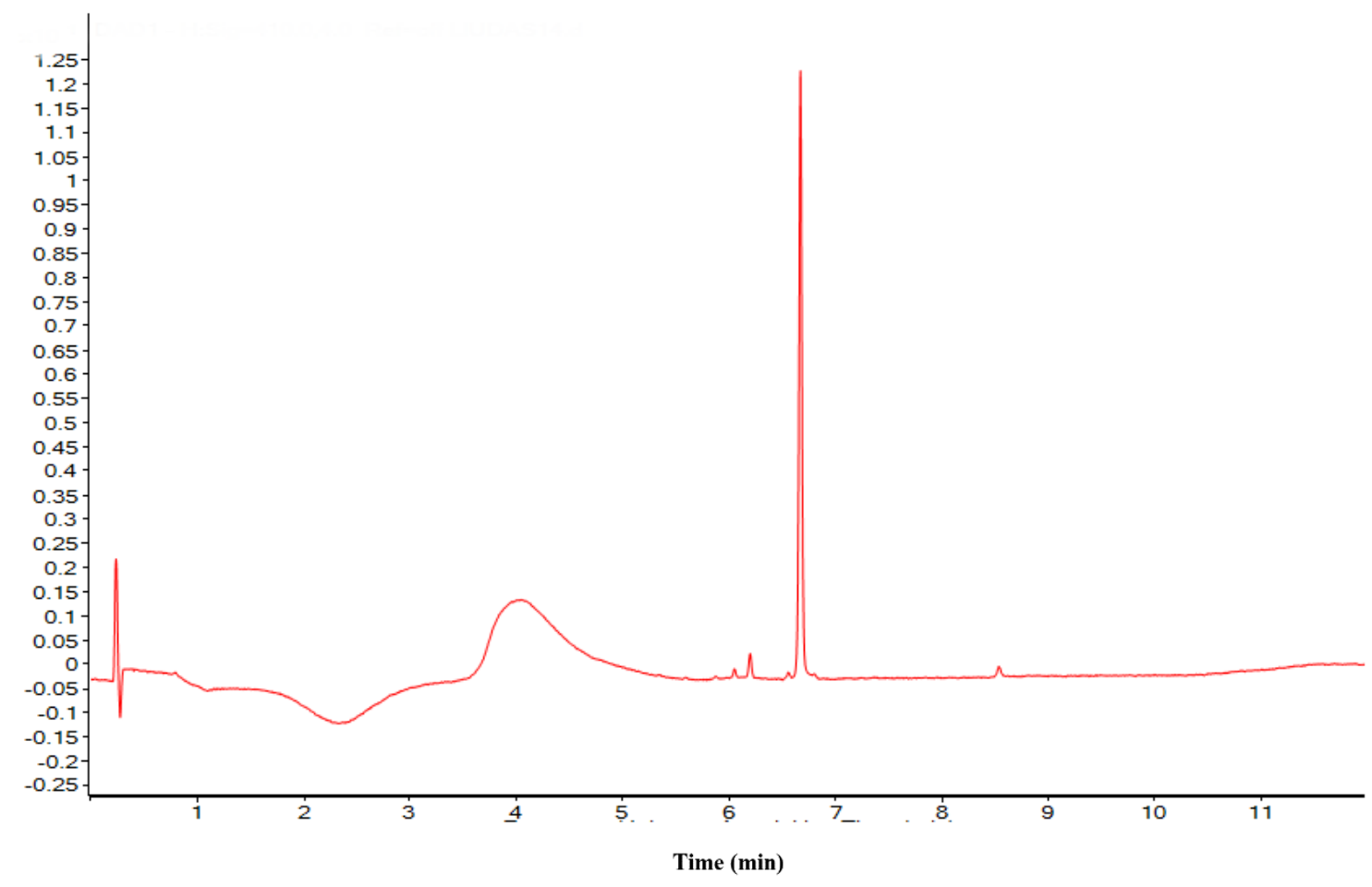

(2R,S)-[(5Z)-5-\{[4-(dimethylamino)naphthalene-1-yl]methylidene $\}-4-0 \times 0-2-t h i o x 0-1,3-$ thiazolidin-3-yl]pentanoic acid (15).

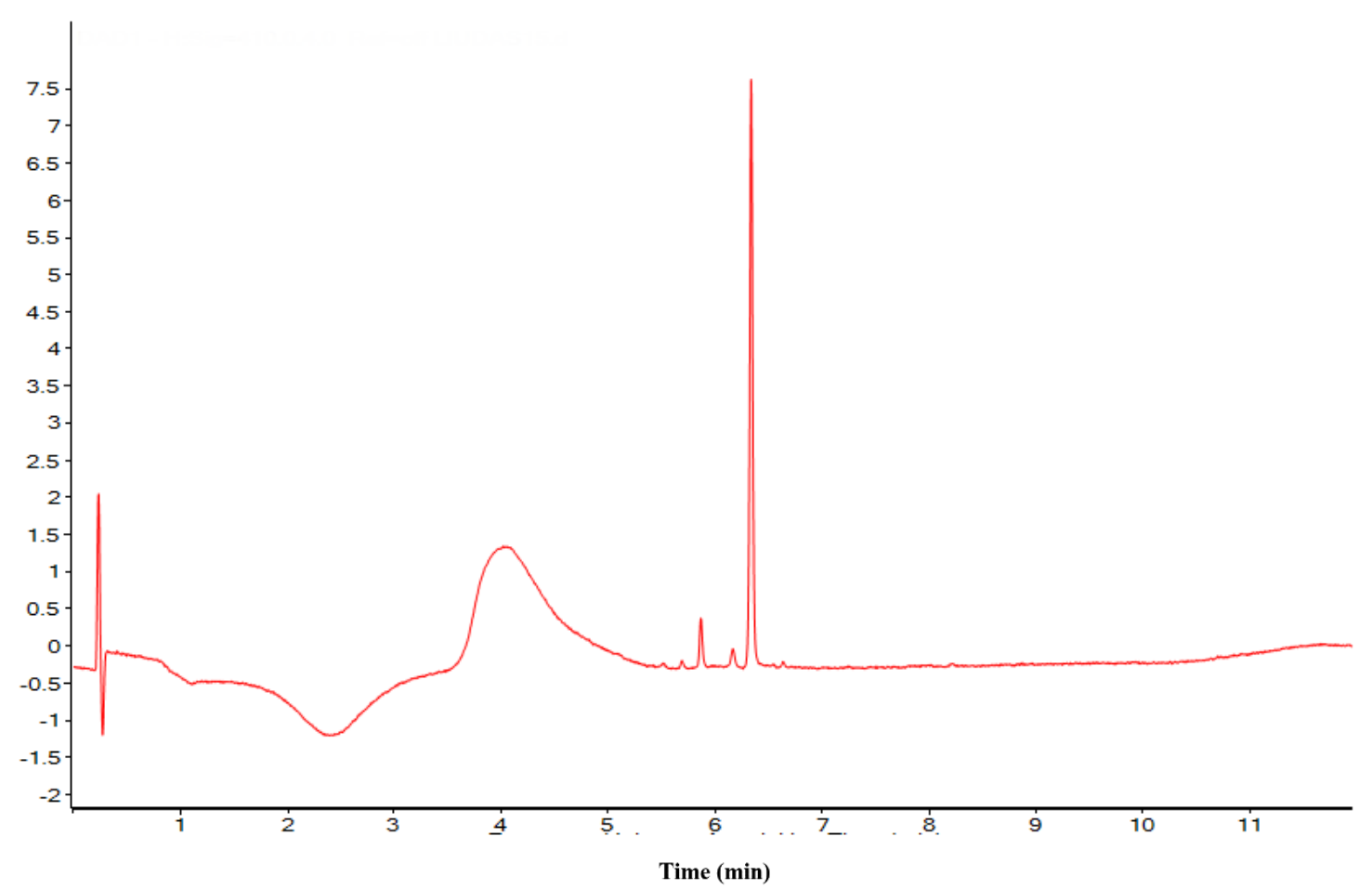


(2R,S)-[(5Z)-5-\{[4-(dimethylamino)naphthalene-1-yl]methylidene\}-4-0xo-2-thioxo-1,3thiazolidin-3-yl]hexanoic acid (16).

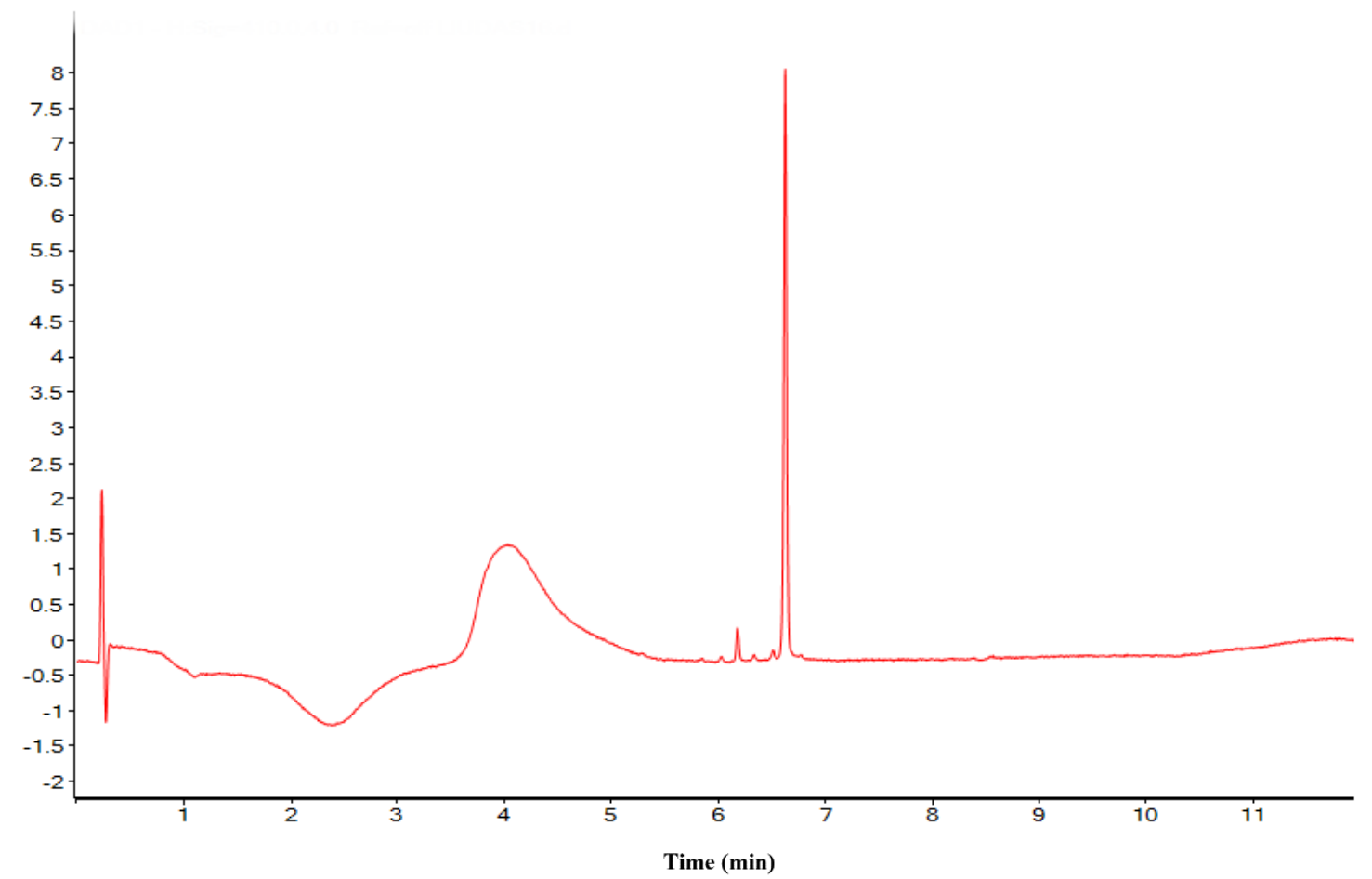

(2S)-3-(1H-indol-3-yl)-2-[(5Z)-5-\{[4-(dimethylamino)naphthalene-1-yl]methylidene $\}-4-0 x 0-$ 2-thioxo-1,3-thiazolidin-3-yl]propanoic acid (17).

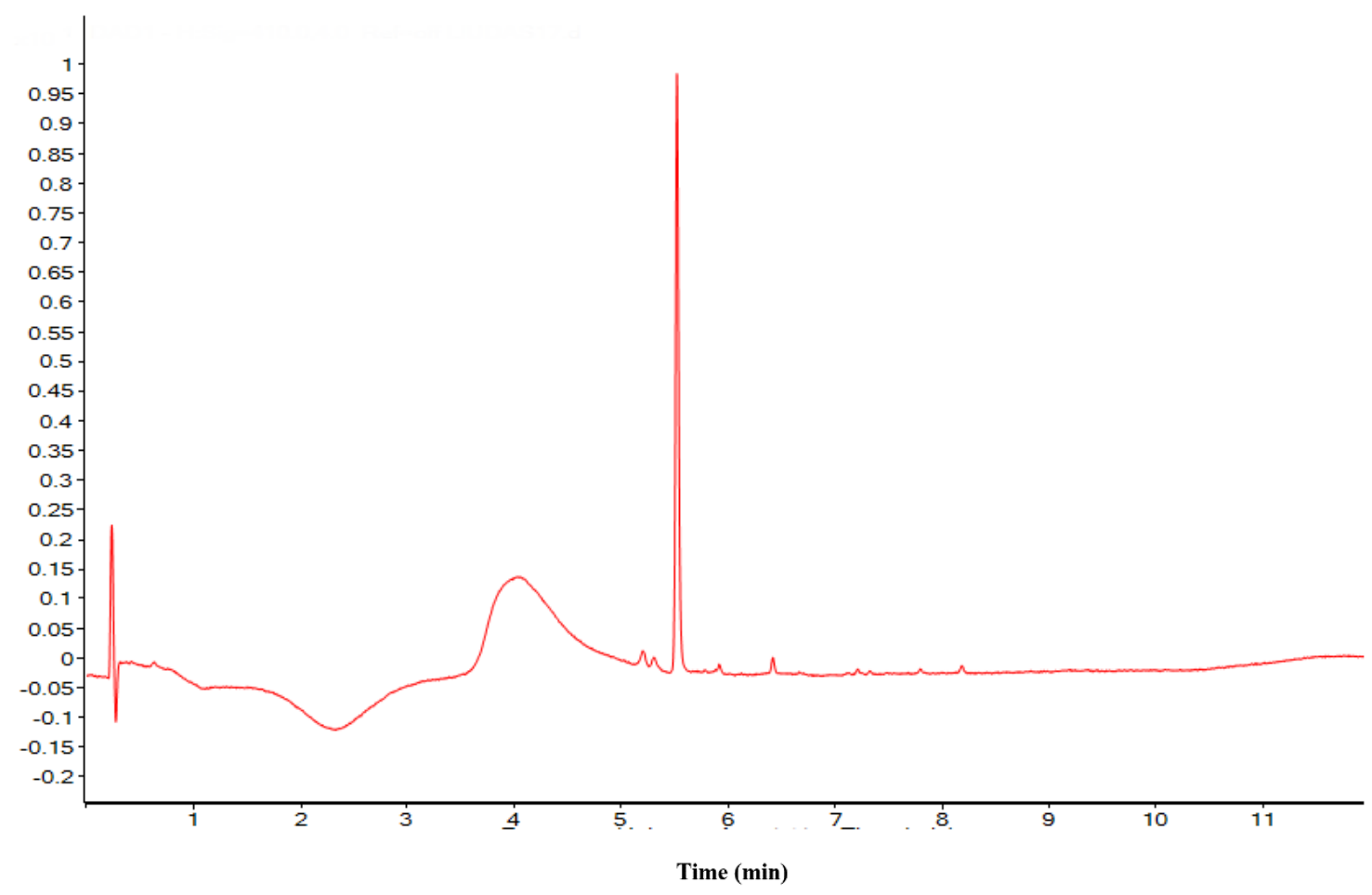


\{3-methyl-1-[(5Z)-5-\{[4-(dimethylamino)naphthalene-1-yl]methylidene\}-4-oxo-2-thioxo-1,3thiazolidin-3-yl]-3-methylbutyl\}phosphonic acid (18).

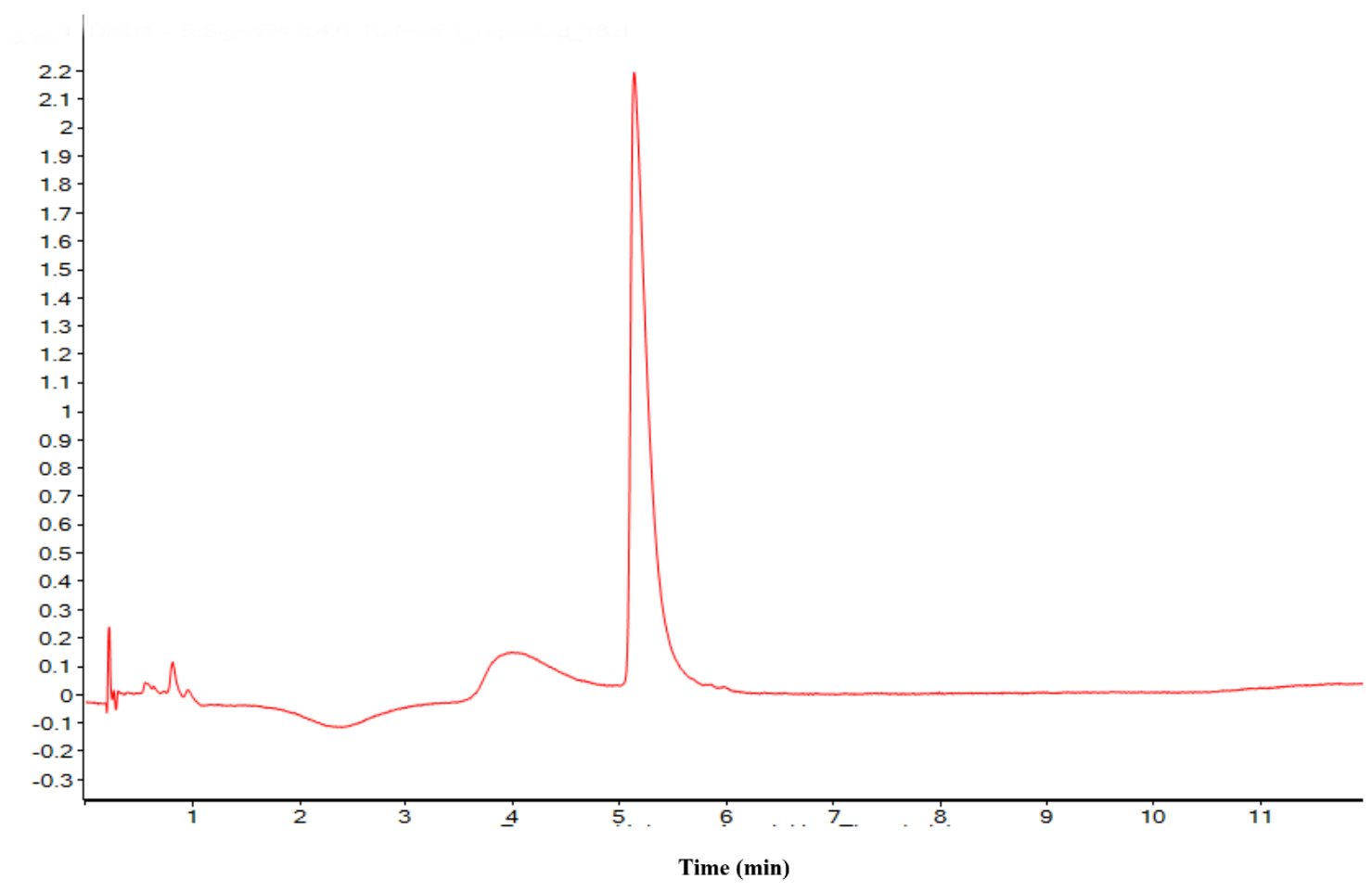

N-\{(5Z)-5-[4-(dimethylamino)benzylidene]-4-oxo-2-thioxo-1,3-thiazolidin-3-yl $\}-3-$ methylbutanamide (19).

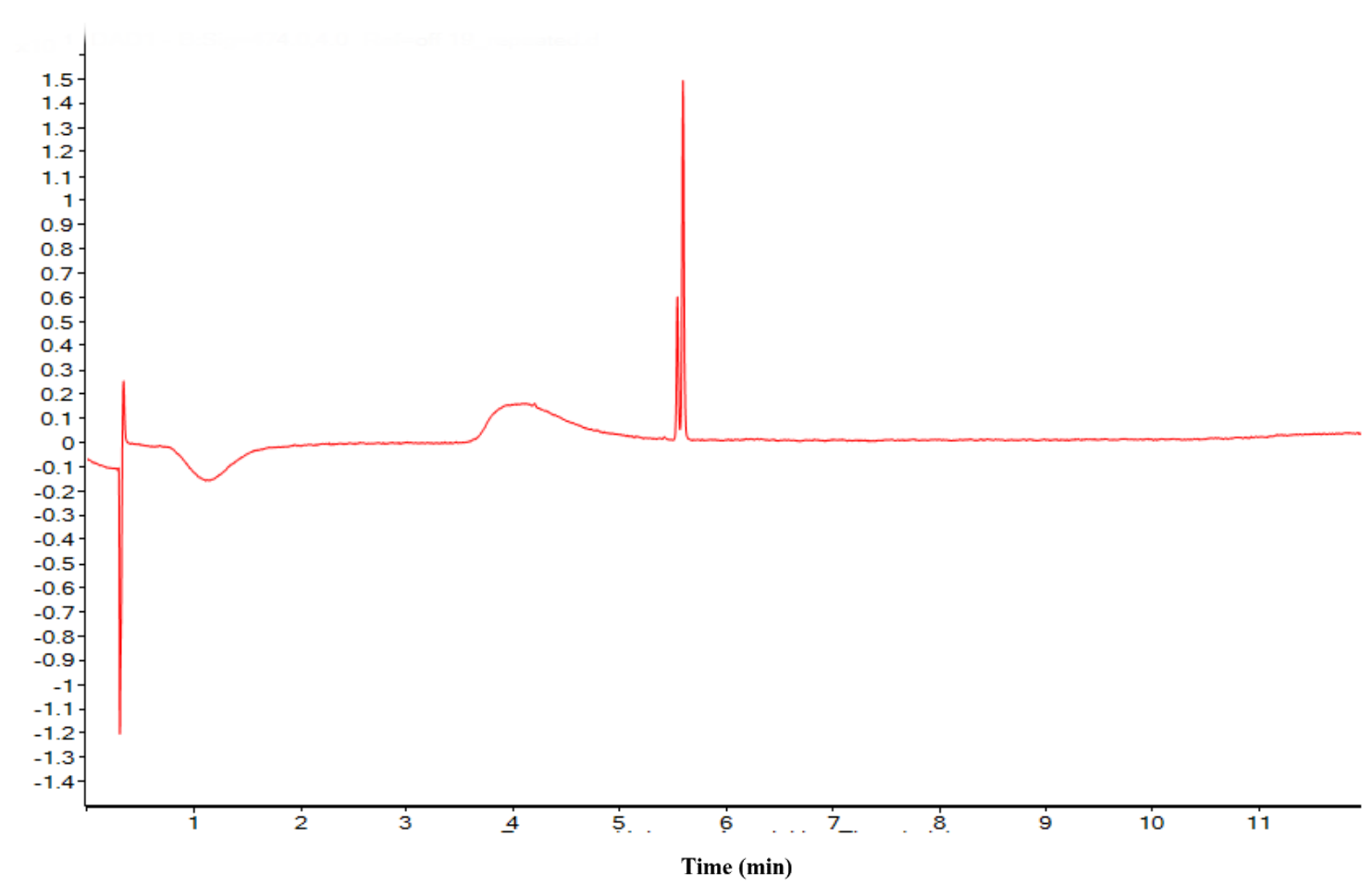


3-\{(5Z)-5-[4-(dimethylamino)benzylidene]-4-oxo-2-thioxo-1,3-thiazolidin-3-yl\}propanoic acid (20).

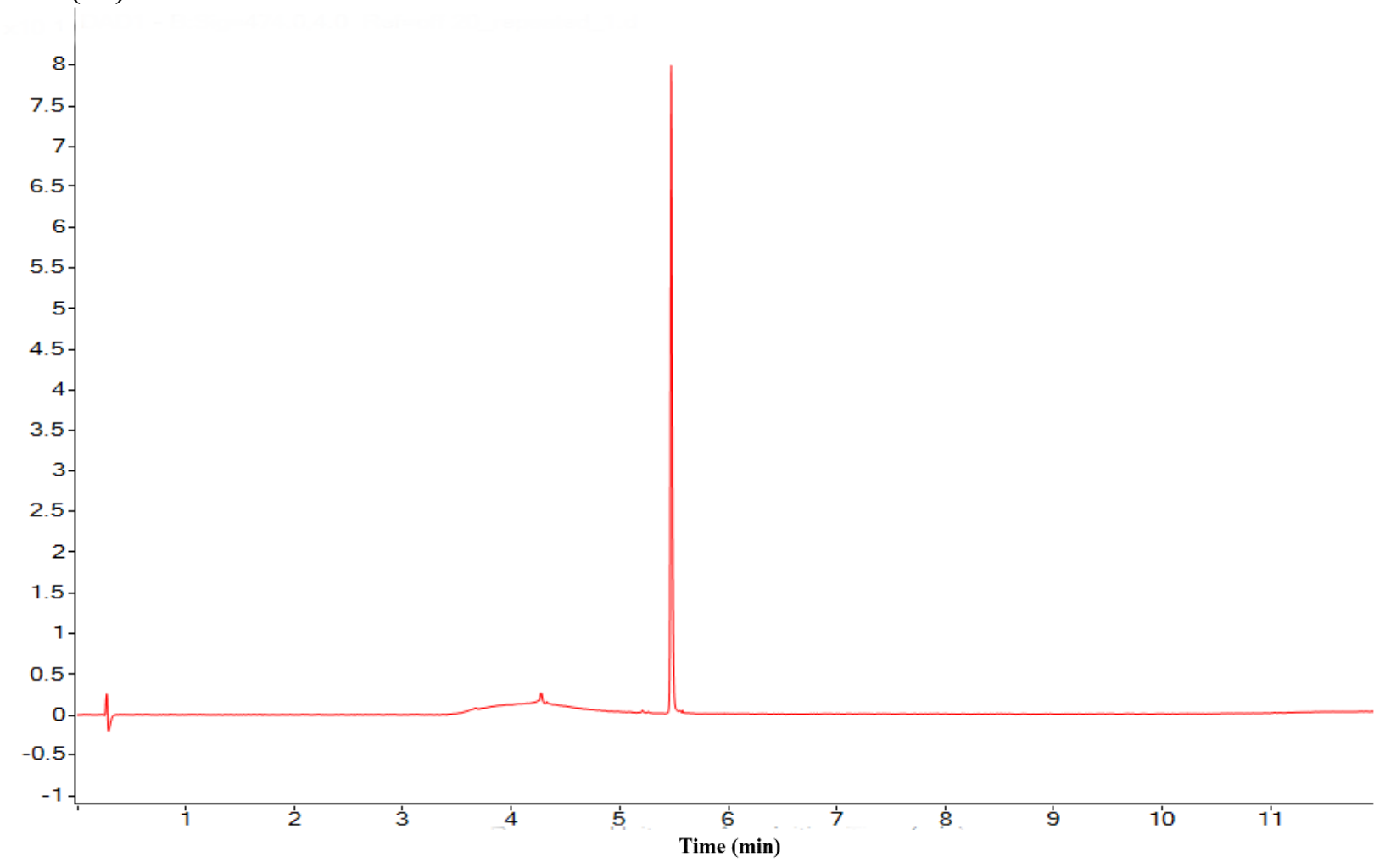

(2S)-2-\{(5Z)-5-[4-(diethylamino)benzylidene]-4-oxo-2-thioxo-1,3-thiazolidin-3-yl\}-4methylpentanoic acid (21).

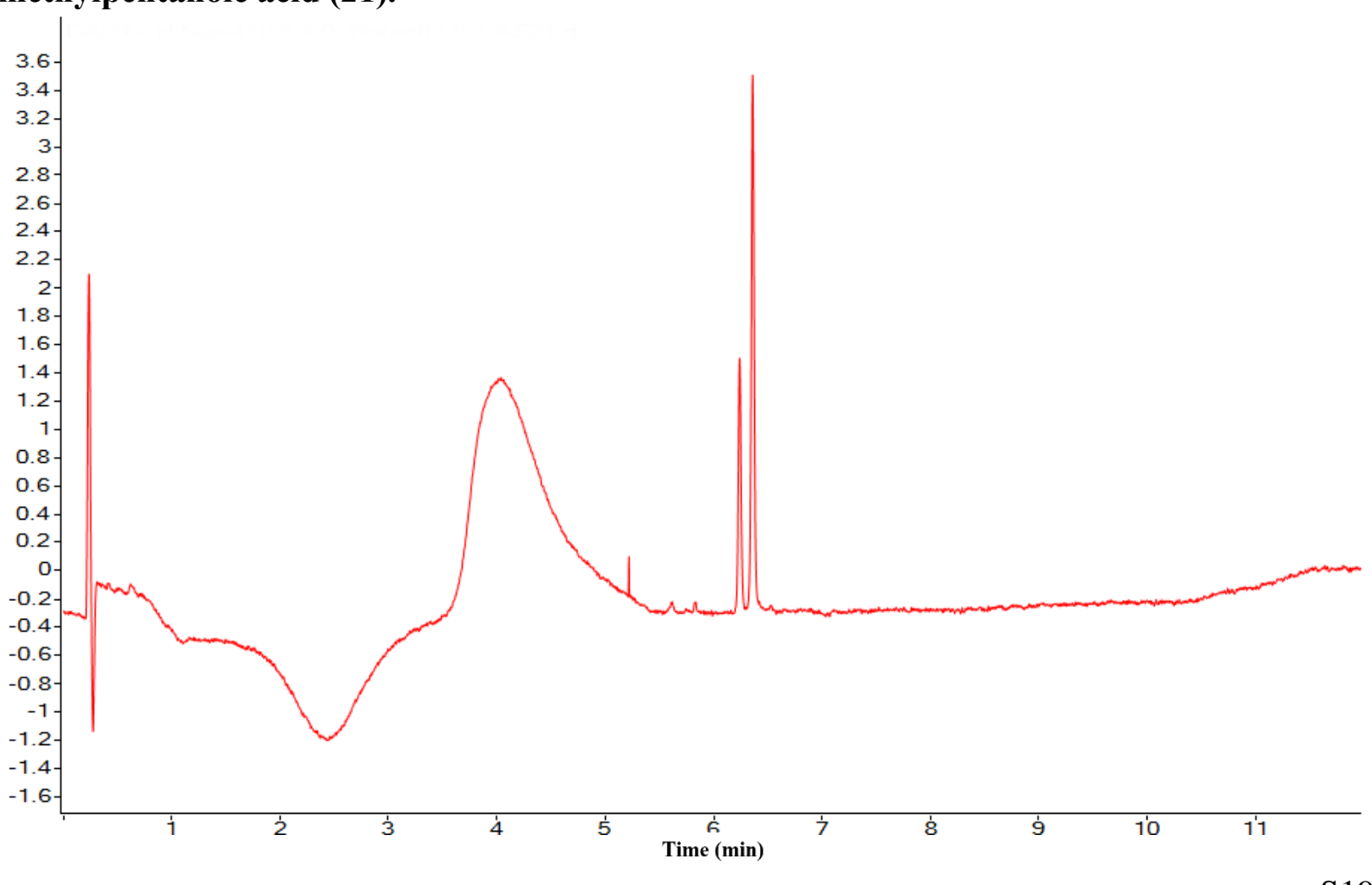


(2S)-2-\{(5Z)-5-[4-(methylsulfanyl)benzylidene]-4-oxo-2-thioxo-1,3-thiazolidin-3-yl\}methyl pentanoic acid (22).

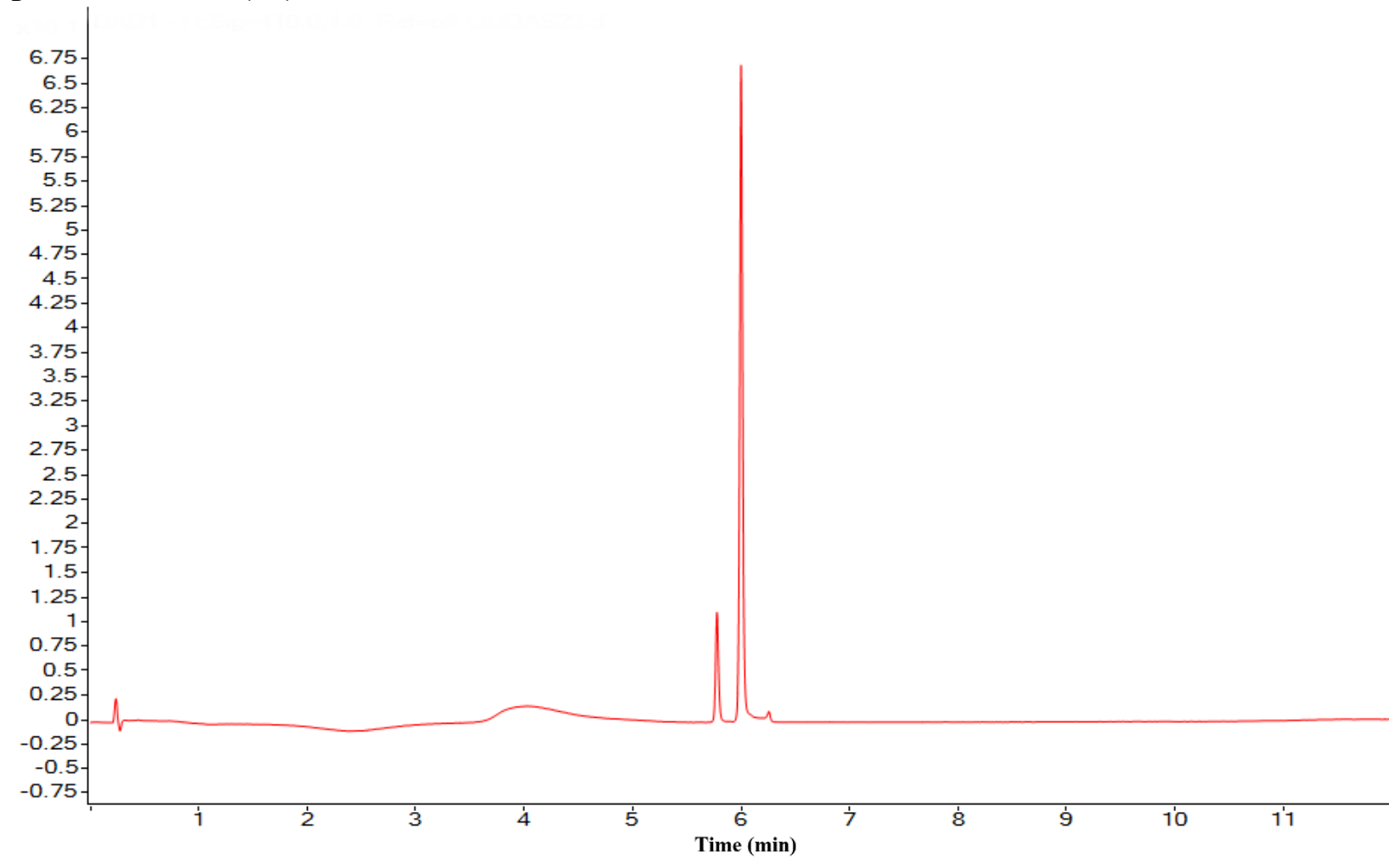

(2S)-2-[(5Z)-5-(4-fluorobenzylidene)-4-oxo-2-thioxo-1,3-thiazolidin-3-yl]-4-ethylpentanoic acid (23).

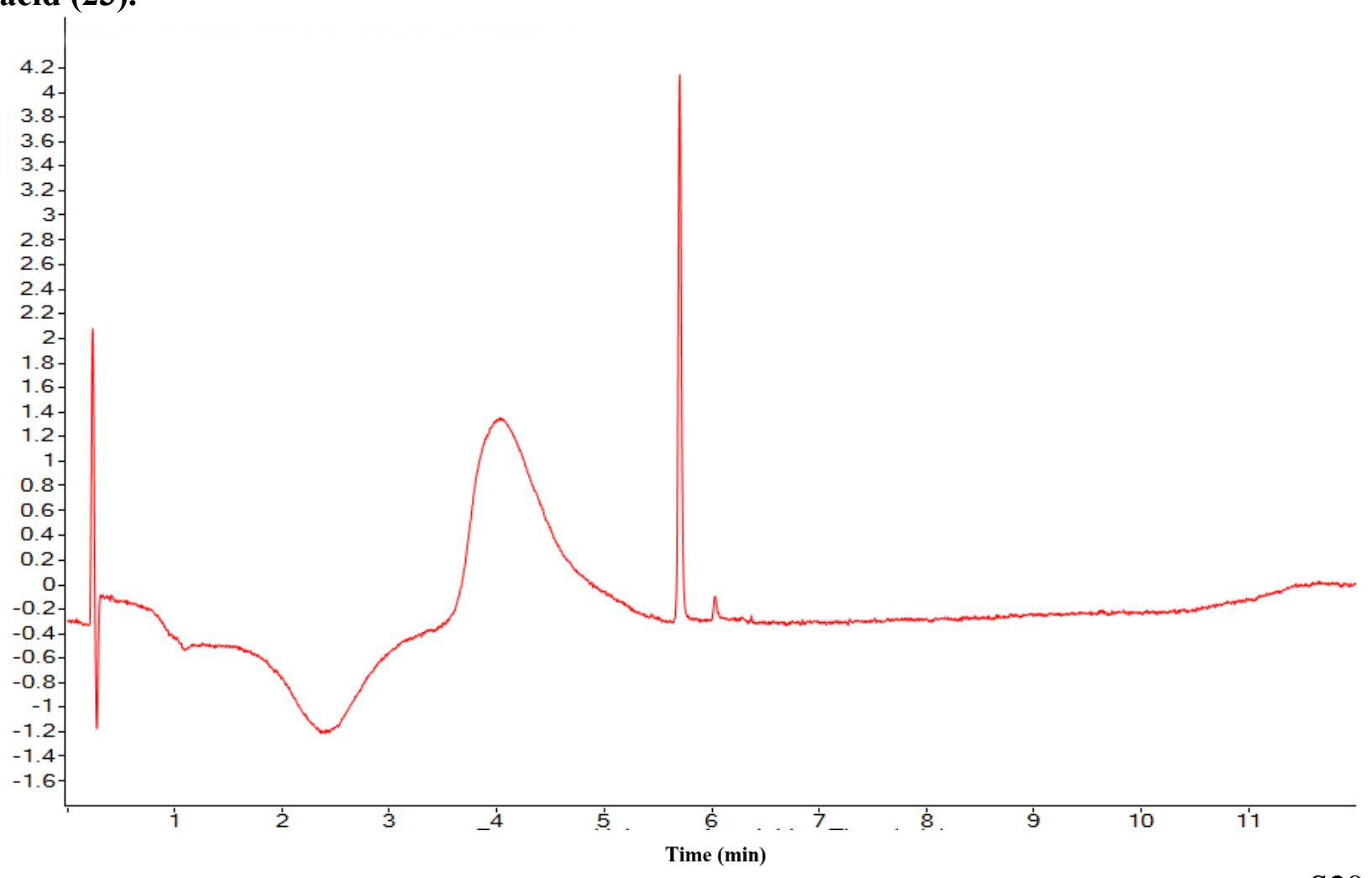


(2S)-2-\{(5Z)-5-[4-(diethylamino)benzylidene]-4-oxo-2-thioxo-1,3-thiazolidin-3-yl]-3-(1Hindol-3-yl)propanoic acid (24).

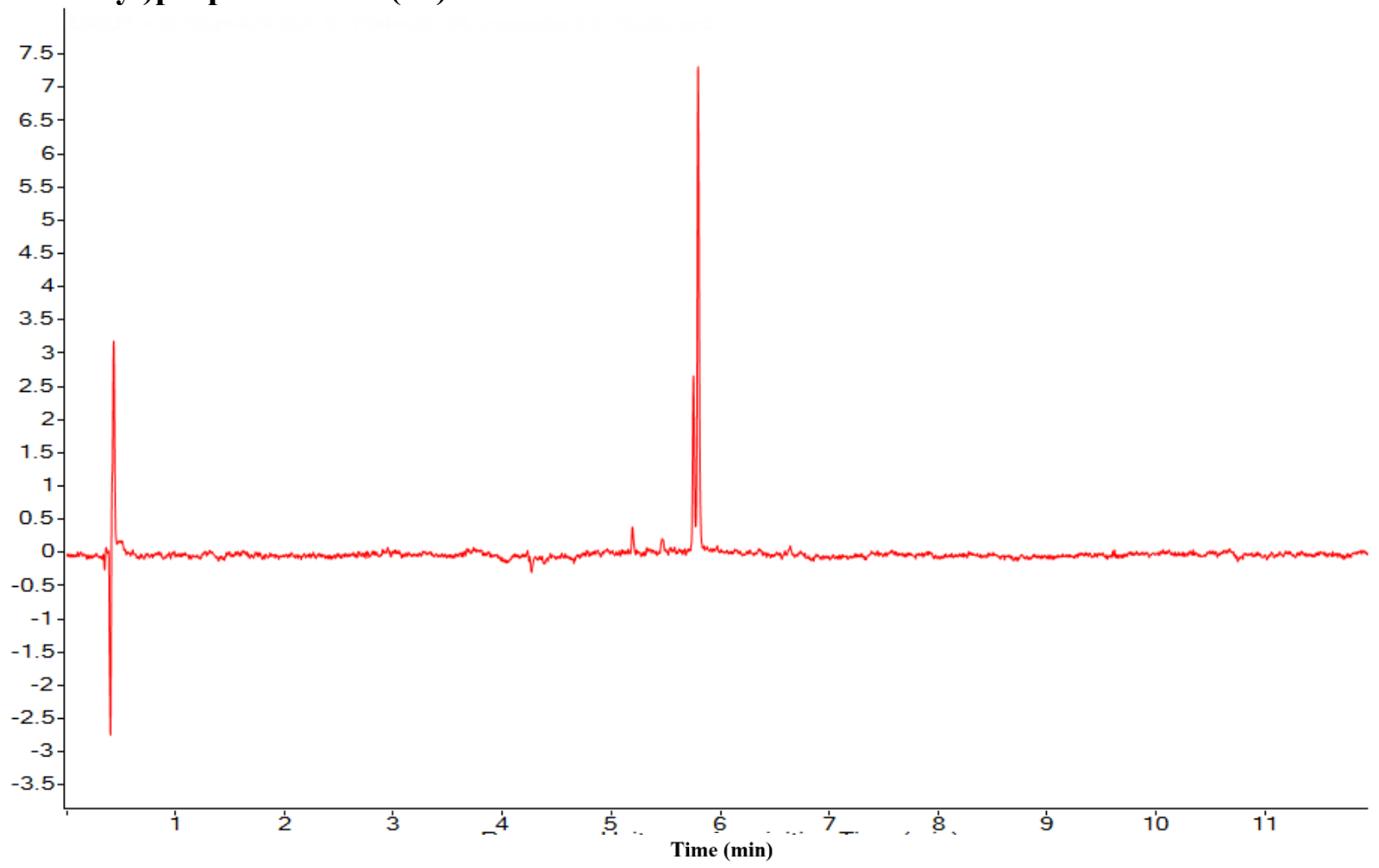

(2S)-2-[(5Z)-5-(4-propylbenzylidene-4-oxo-2-thioxo-1,3-thiazolidin-3-yl]-3-(1H-indol-3yl)propanoic acid (25).

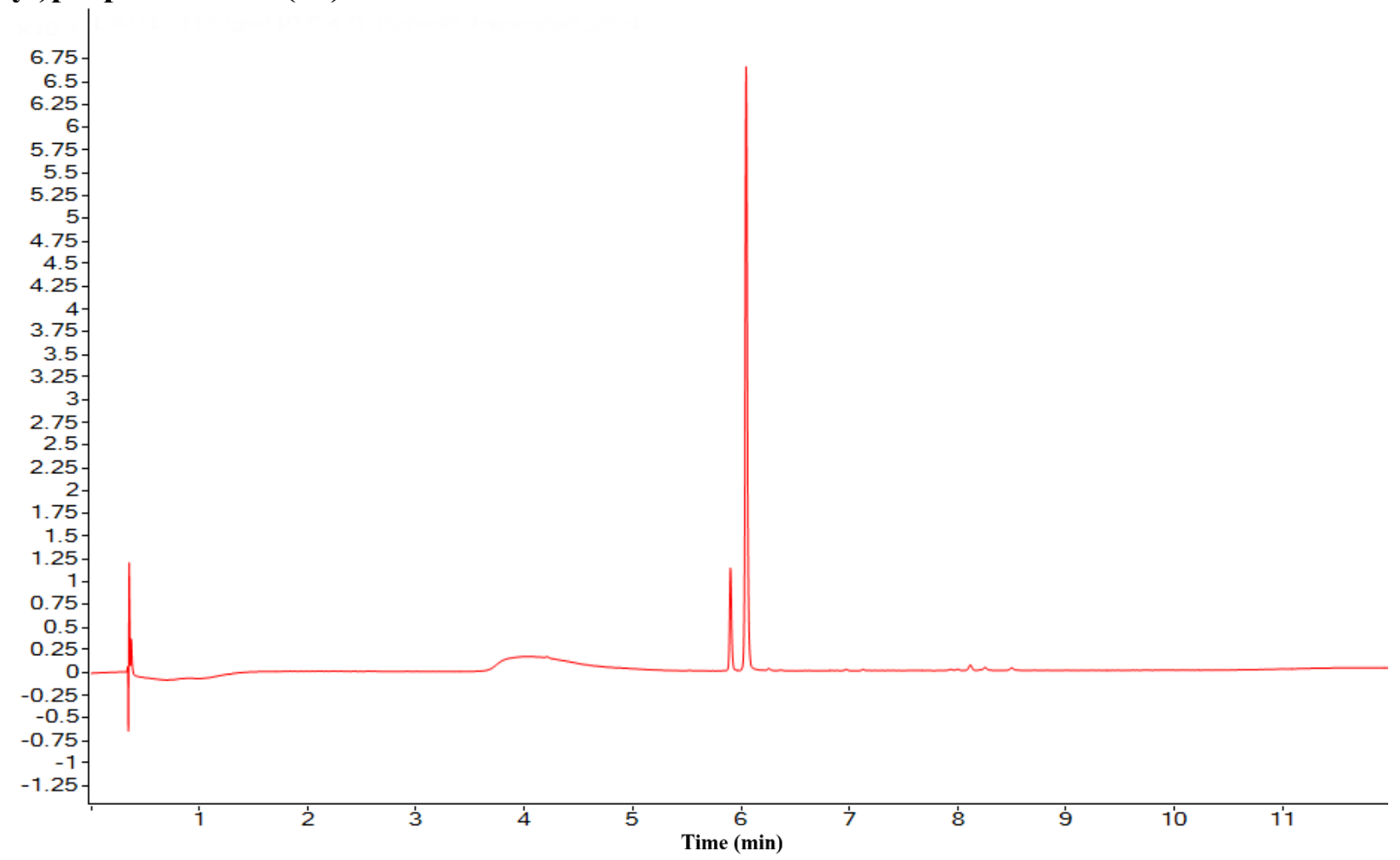


(2S)-2-[(5Z)-5-\{4-[bis(2-chloroethyl)amino]benzylidene-4-oxo-2-thioxo-1,3-thiazolidin-3-yl]3-(1H-indol-3-yl)propanoic acid (26).

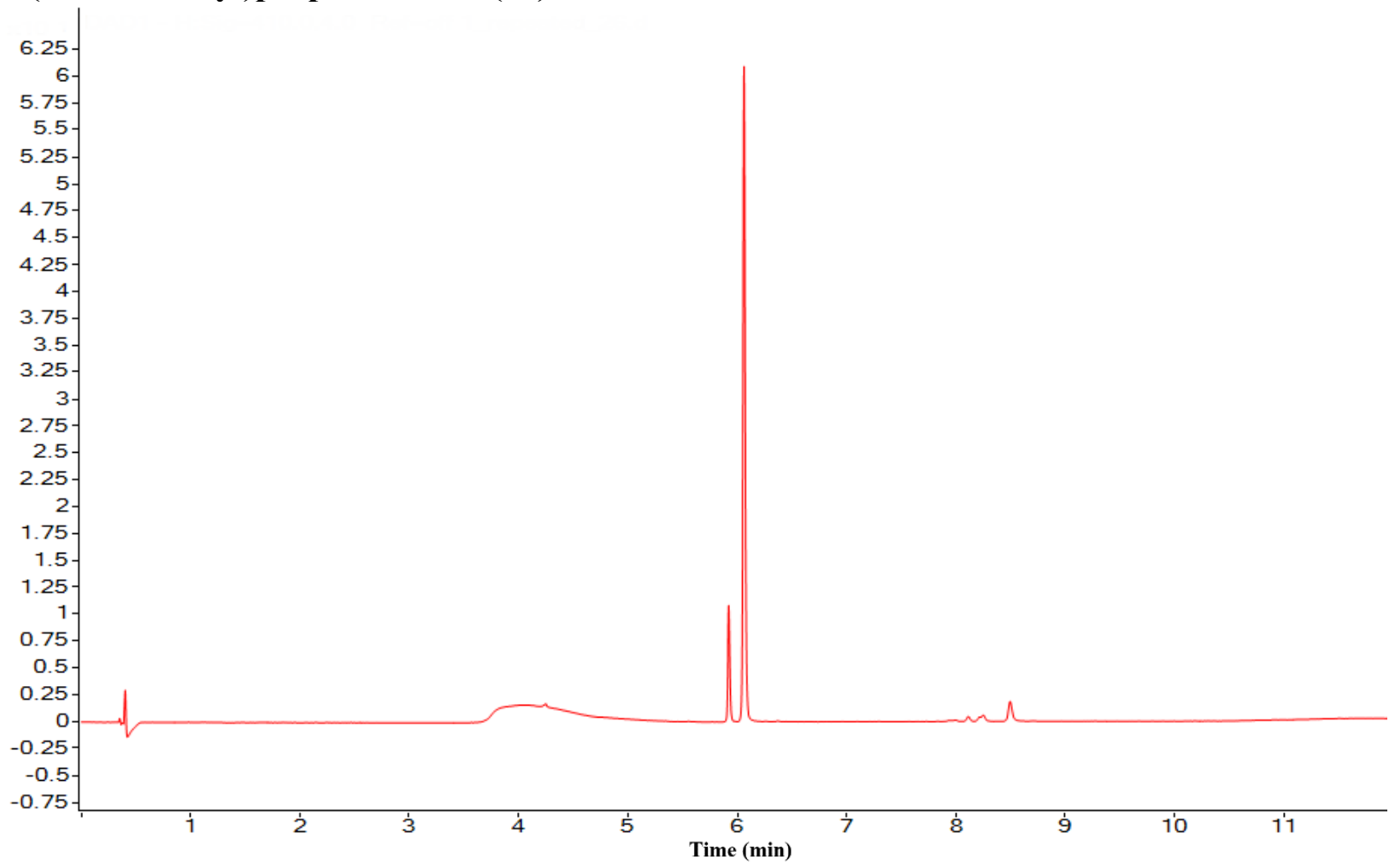

(2R)-2-[(5Z)-4-oxo-5-(4-propylbenzylidene-4-oxo-2-thioxo-1,3-thiazolidin-3-yl]-3-(1H-indol3-yl)propanoic acid (27).

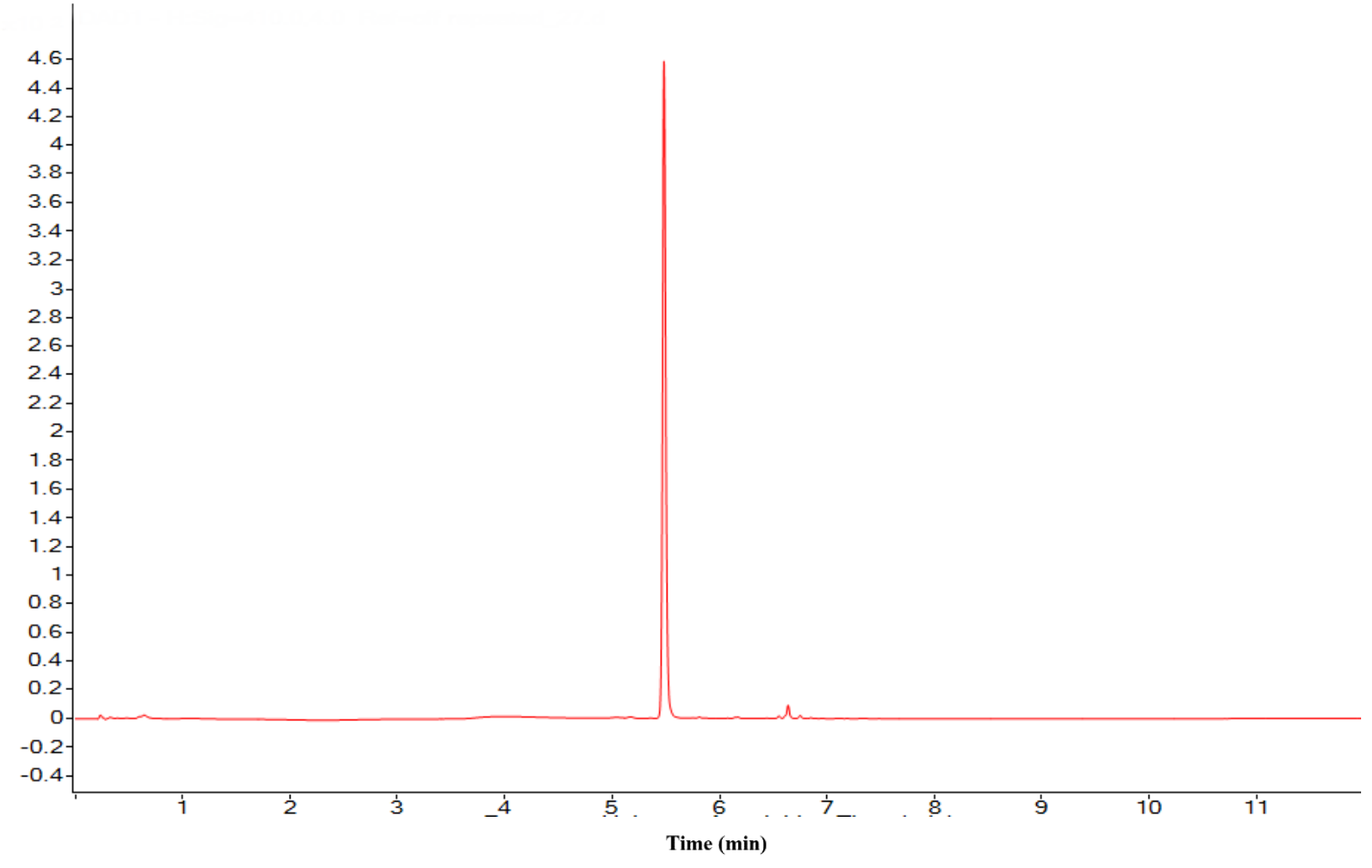


(2R)-2-[(5Z)-5-\{4-[bis(2-chloroethyl)amino]benzylidene-4-oxo-2-thioxo-1,3-thiazolidin-3yl]-3-(1H-indol-3-yl)propanoic acid (28).

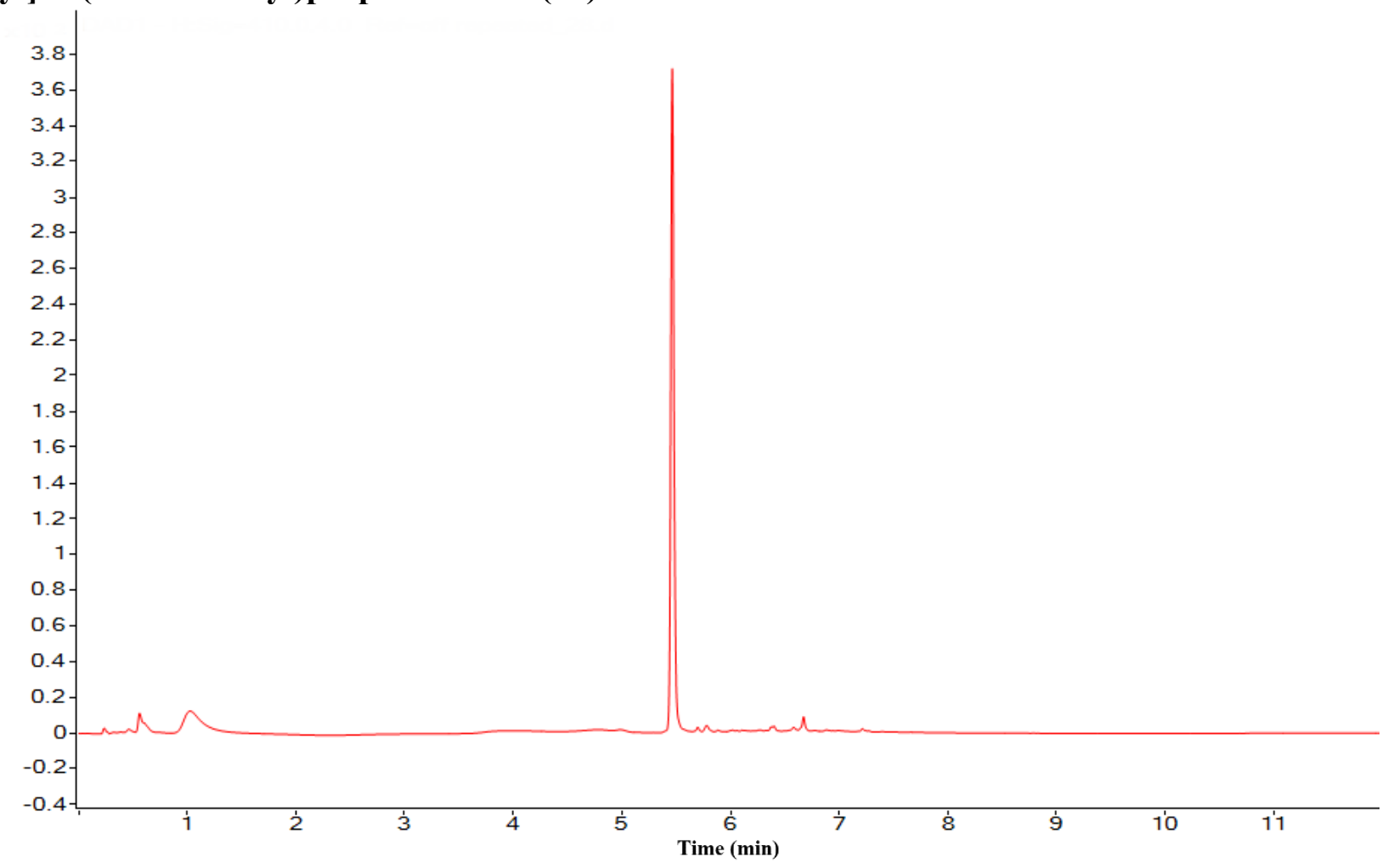

(2S)-[(5Z)-5-(4-chlorobenzylidene)-4-oxo-2-thioxo-1,3-thiazolidin-3-yl]-3-(1H-indol-3yl)propanoic acid (29).

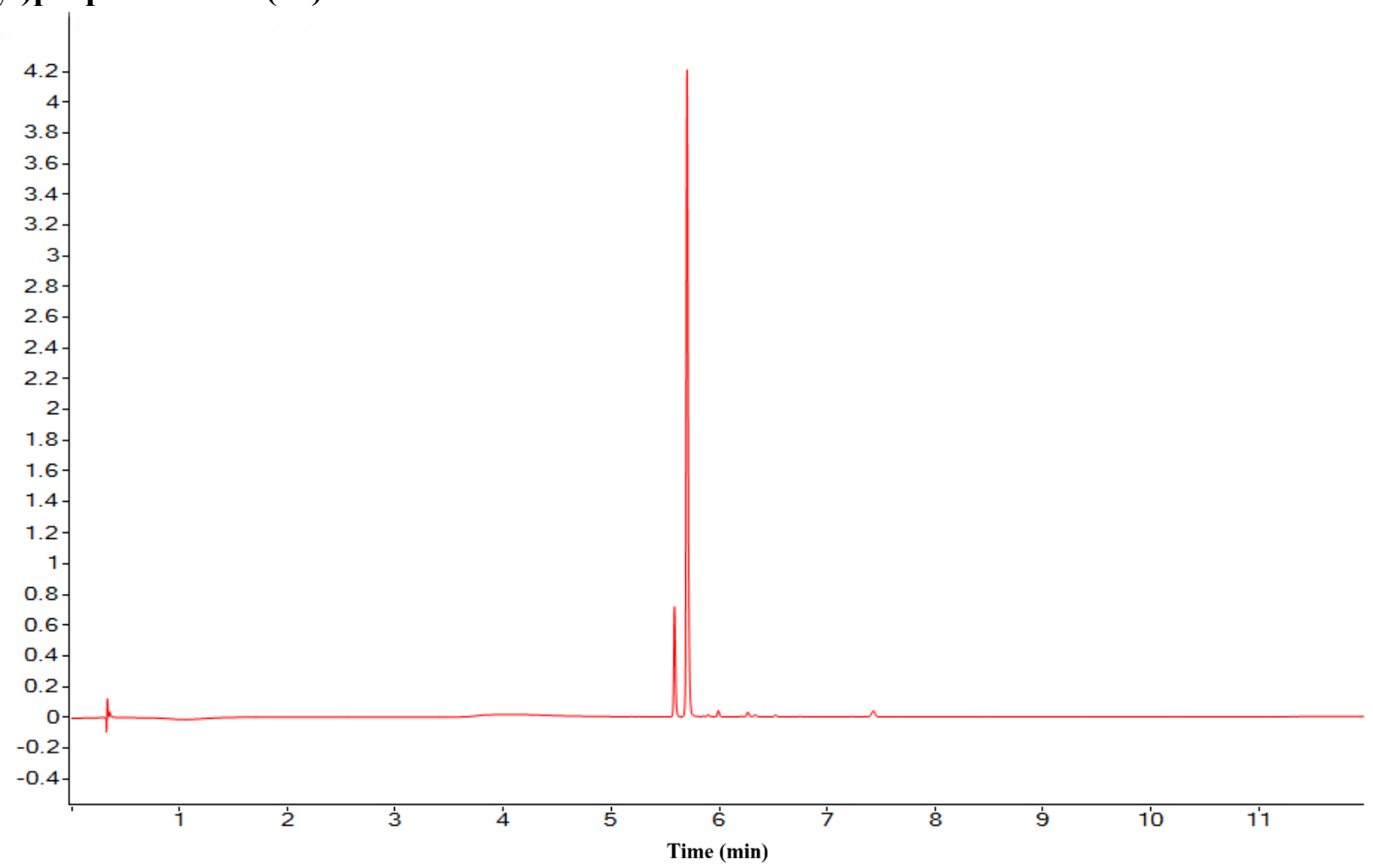


(2S)-[(5Z)-5-(2,4-dichlorobenzylidene)-4-oxo-2-thioxo-1,3-thiazolidin-3-yl]-3-(1H-indol-3yl)propanoic acid (30).

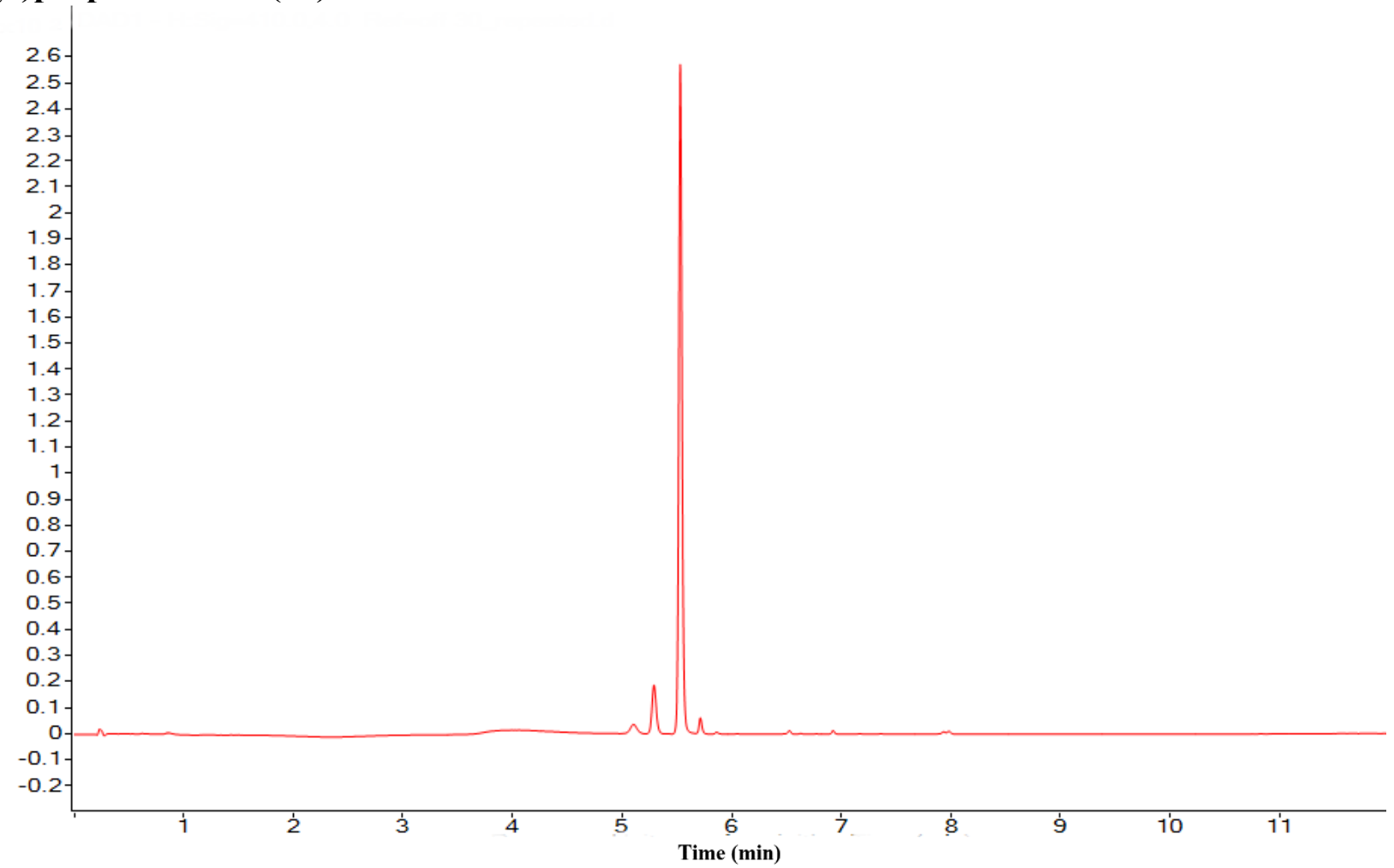

(5Z)-5-[4-(diethylamino)benzylidene]-3-[2-(1H-indol-3-yl)ethyl]-2-thioxo-1,3-thiazolidin-4one (31).

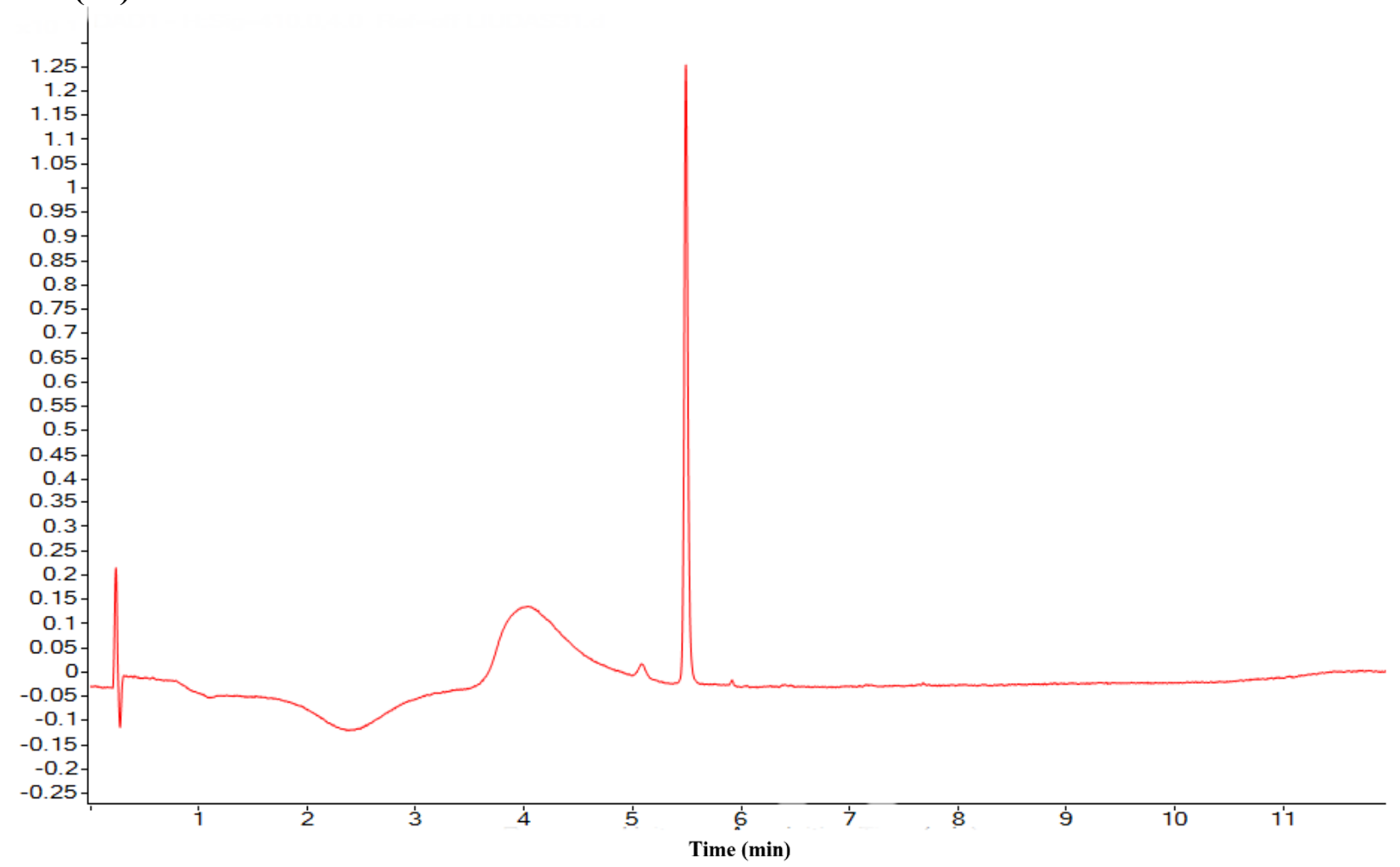


(2S)-2-\{(5Z)-5-[(10-chloroanthracen-9-yl)methylidene-4-oxo-2-thioxo-1,3-thiazolidin-3-yl]3-(1Hindol-3-yl)propanoic acid (32).

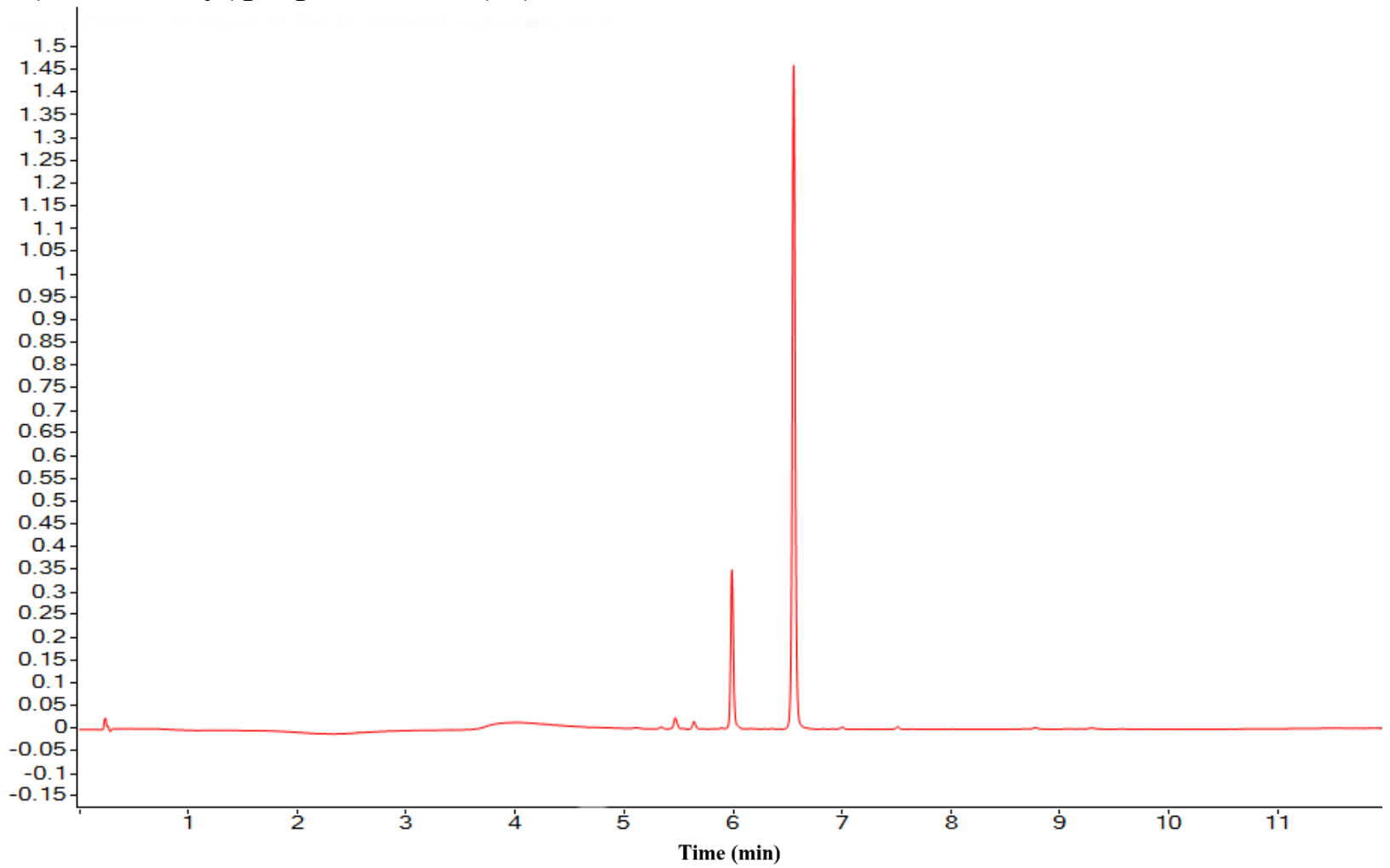

(2S)-2-\{(5Z)-5-[(4-methylnaphthalen-1-yl)methylidene-4-oxo-2-thioxo-1,3-thiazolidin-3-yl]3-(1Hindol-3-yl)propanoic acid (33).

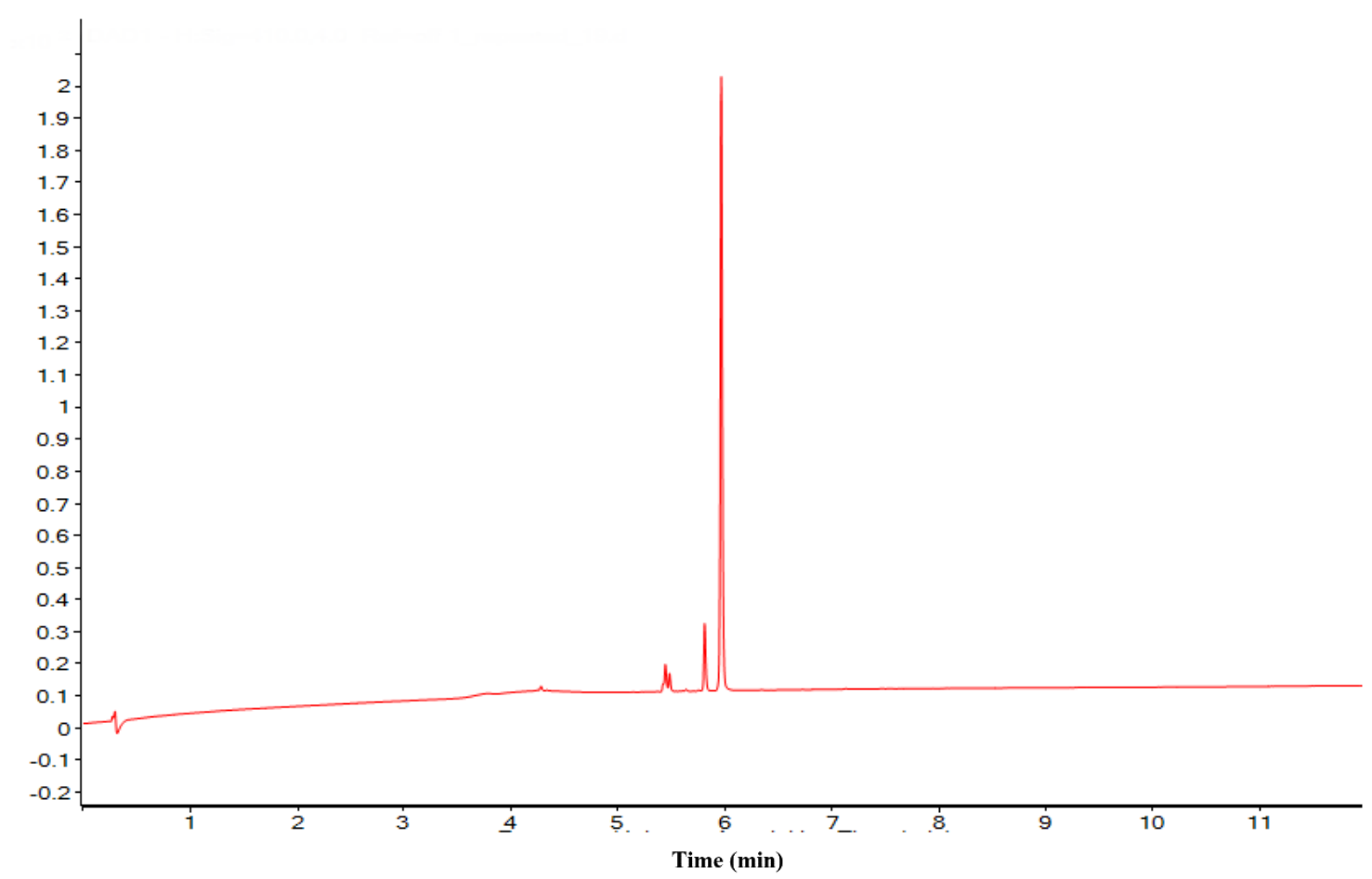


(2R)-2-\{(5Z)-5-[(4-methylnaphthalen-1-yl)methylidene-4-oxo-2-thioxo-1,3-thiazolidin-3-yl]3-(1H-indol-3-yl)propanoic acid (34).

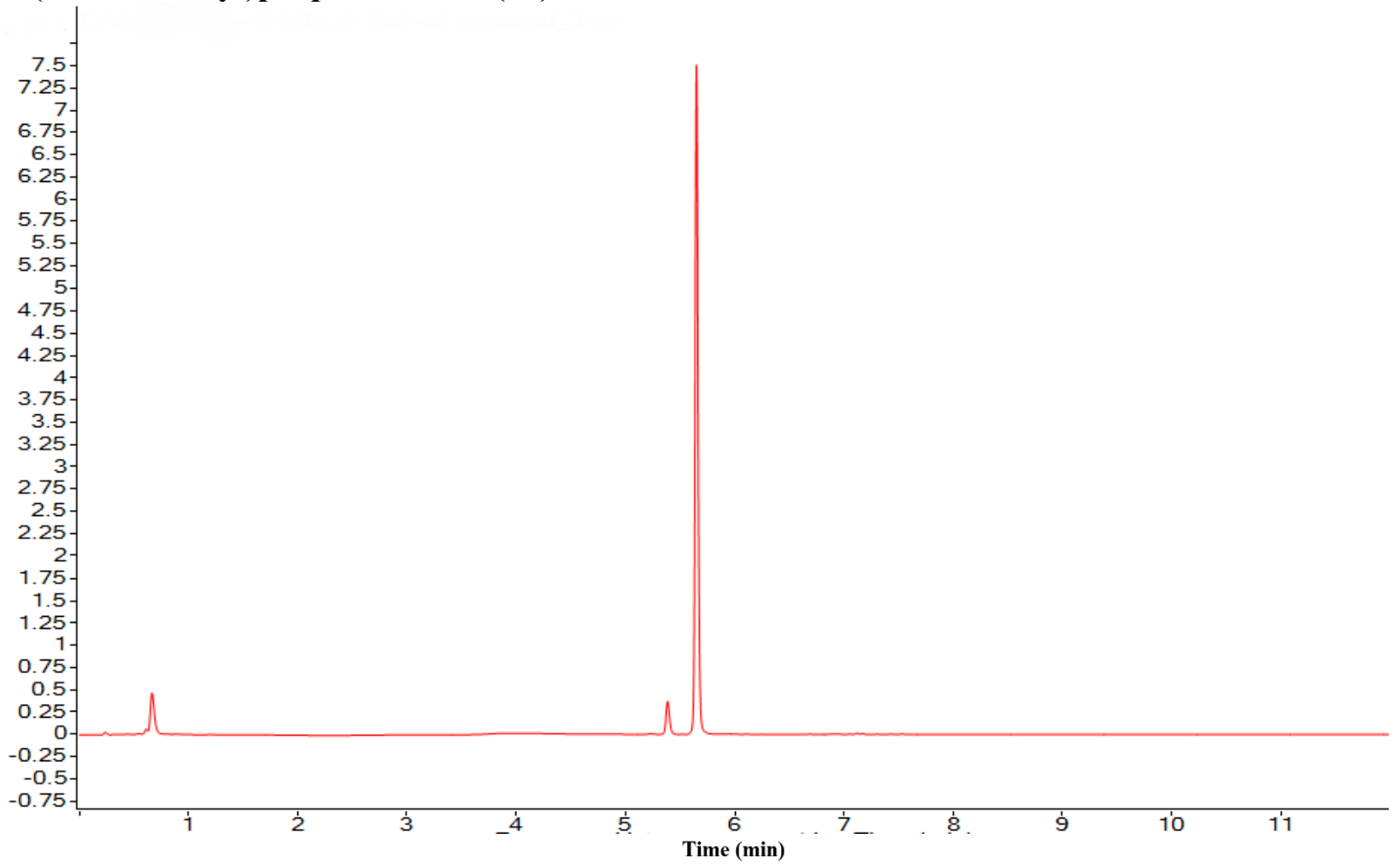




\section{References SI}

1. Sullivan, T. J.; Truglio, J. J.; Boyne, M. E.; Novichenok, P.; Zhang, X.; Stratton, C. F.; Li, H. J.; Kaur, T.; Amin, A.; Johnson, F.; Slayden, R. A.; Kisker, C.; Tonge, P. J., High affinity InhA inhibitors with activity against drug-resistant strains of Mycobacterium tuberculosis. $A C S$ chemical biology 2006, 1 (1), 43-53. 